\title{
The impact of common envelope development criteria on the formation of LIGO/Virgo sources.
}

\author{
A. Olejak ${ }^{1}$ K. Belczynski ${ }^{1}$ N. Ivanova ${ }^{2}$ \\ ${ }^{1}$ Nicolaus Copernicus Astronomical Center, Polish Academy of Sciences, ul. Bartycka 18, 00-716 Warsaw, Poland \\ e-mail: aolejak@camk.edu.pl,chrisbelczynski@gmail.com \\ 2 Department of Physics, University of Alberta, Edmonton, AB, T6G 2E7, Canada
}

Received ; accepted

\begin{abstract}
The treatment and criteria for development of unstable Roche lobe overflow (RLOF) that leads to the common envelope (CE) phase have hindered the evolutionary predictions for decades. In particular, the formation of black hole-black hole (BH-BH), black holeneutron star (BH-NS), and neutron star-neutron star (NS-NS) merging binaries depends sensitively on the CE phase in classical isolated binary evolution model. All these mergers are now reported as LIGO/Virgo sources or source candidates. CE is even considered by some as a mandatory phase in the formation of BH-BH, BH-NS or NS-NS mergers in binary evolution models. At the moment, there is no full first-principles model for development of CE. We employ the StarTrack population synthesis code to test the current advancements in studies on stability of RLOF for massive donors to assess their effect on LIGO/Virgo source population. In particular, we allow for more restrictive CE development criteria for massive donors $\left(M>18 M_{\odot}\right)$. We also test a modified condition for switching between different types of stable mass transfer, thermal or nuclear timescale. Implemented modifications significantly influence basic properties of merging double compact objects, sometimes in non-intuitive way. For one of tested models with restricted $\mathrm{CE}$ development criteria local merger rate density for $\mathrm{BH}-\mathrm{BH}$ systems increased by a factor of 2-3 due to emergence of a new dominant formation scenario without any CE phase. We find that the changes in highly uncertain assumptions on RLOF physics may significantly affect (i) local merger rate density, (ii) shape of the mass and mass ratio distributions, and (iii) dominant evolutionary formation (with and without CE) scenarios of LIGO/Virgo sources. Our results demonstrate that without sufficiently strong constraints on RLOF physics, one is not able to draw fully reliable conclusions about the population of double compact object systems based on population synthesis studies.
\end{abstract}

Key words. stellar evolution - close binary systems - common envelope - stability of RLOF

\section{Introduction}

The concept of close binary systems' formation, such as merging double compact objects (DCO) or X-ray binaries, through the common envelope (CE) phase, begun to appear in the literature almost 50 years ago (Paczynski 1976; van den Heuvel 1976). In this concept, one binary component enters the other binary companion's envelope. The orbital energy is transferred to the envelope due to various drag forces, resulting in the binary orbit shrinking. Finally, the envelope either can be ejected from the system, leaving behind a newly formed close binary system, or the two stars merge. One of the approaches to find the outcome of a CE event without doing detailed 3D simulations is by considering the energy budget of a $\mathrm{CE}$ event and hence is known as energy formalism. In its parameterized form, it has been introduced by Webbink (1984) and Livio \& Soker (1988), and is known as " $\alpha_{\mathrm{CE}}$-formalism". Due to its simplicity, $\alpha_{\mathrm{CE}}$ - formalism is still widely used in population synthesis studies (e.g., Vigna-Gómez et al. 2020). This simplified form of energy formalism equates only two energies: the envelope's binding energy (the energy required to eject the envelope to infinity) and the change in the orbital energy (the available energy source).
This allows to estimate the orbital separation after a CE event, assuming that envelope has been ejected:

$\frac{G M_{\mathrm{don}, \mathrm{i}} M_{\mathrm{don}, \mathrm{env}}}{\lambda R_{\mathrm{don}, \mathrm{lob}}}=\alpha_{\mathrm{CE}}\left(\frac{G M_{\mathrm{don}, \mathrm{f}} M_{\mathrm{comp}, \mathrm{f}}}{2 a_{\mathrm{f}}}-\frac{G M_{\mathrm{don}, \mathrm{i}} M_{\mathrm{comp}, \mathrm{i}}}{2 a_{\mathrm{i}}}\right)$,

Here $a_{\mathrm{i} / \mathrm{f}}-$ initial or final orbital separation, $M_{\mathrm{don}, \mathrm{i} / \mathrm{f}}-$ initial or final donor mass, $M_{\text {comp, } / \text { f }}-$ initial or final companion mass respectively, $G$ - gravitational constant, $M_{\text {don,env }}-$ mass of the donor envelope, $R_{\text {don,lob }}$ - Roche lobe radius of the donor at the onset of Roche-lobe Overflow (RLOF), $i$ and $f$ - initial and final values of mass and separation, and $\lambda$ - is a measure of the donor central concentration (de Kool 1990; Dewi \& Tauris 2000; Xu \& Li 2010).

Equation 1 postulates that the transfer of binary system orbital energy into the energy of the envelope takes place with some efficiency $\alpha_{\mathrm{CE}}$, which, unless other energy sources are present, can not be more than one. Simulations and observations of $\mathrm{CE}$ phase indicates that the value of parameter is typically $\alpha_{\mathrm{CE}}<0.6-1.0$ (Zuo \& Li 2014, Nandez \& Ivanova 2016, Clayton et al. 2017; Iaconi \& De Marco 2019). On the other hand, if the simulations of a CE event are performed while including more physical processes, for example, accretion, the effective value of $\alpha_{\mathrm{CE}}$ can be as high as 5 (e.g., see Fragos et al. 
2019). A similar effect of increasing the apparent CE efficiency to more than one be produced by exotic nucleosynthesis and jets (Podsiadlowski et al. 2010; Shiber et al. 2019; Zevin et al. 2020, Grichener \& Soker 2021). Some population synthesis studies have already adopted such high values (e.g., see Santoliquido et al.2020).

The CE efficiency parameter $\alpha_{\mathrm{CE}}$ and the binding energy parameter $\lambda$ are often coupled in population synthesis studies or in observations' analysis when energy formalism is applied. At the same time, each of them is subject to many uncertainties. For example, $\lambda$ was introduced to relate the "true" binding energy of the envelope (as obtainable from detailed stellar models) to its simple parameterized form. However, what is the true binding energy of the donor, is still the subject of discussion (e.g., see $\S 3$ in Ivanova et al. 2020). The CE efficiency parameter is expected to depend on the system's specifics and which physical processes of energy creation or energy loss took place (e.g., on the system's mass ratio, the evolutionary stage of the donor, or the companion's nature). Derived from observations, values of $\alpha_{\mathrm{CE}}$ seem to be systematically lower for asymptotic giant branch (AGB) donors than for red giant branch (RGB) donors (Iaconi \& De Marco 2019), which have different internal structure and envelope binding energy. AGB donors envelopes are considered to be less tightly bound to the core than those of a RGB donors (Paczyński \& Ziółkowski 1968, Han et al. 1994). However, even taking into account this AGB stars feature does not sufficiently help in successive envelope ejection (Sand et al. 2020). Numerical simulations encounter difficulties in successful envelope ejection unless some other energetic process (except orbital energy release) is included (e.g., Passy et al. 2012; Iaconi et al. 2017, Sand et al. 2020). Also recent studies of Klencki et al. (2021) confirmed that even with the most favorable assumptions, a successful $\mathrm{CE}$ ejection in $\mathrm{BH}$ binaries is only possible if the donor is a massive convective-envelope giant. On the other hand, massive stars (BH progenitors) may be a subject of extensive mass loss through enhanced winds before they reach the RSG stage what may even cause the spontaneous envelope loss (e.g., Vanbeveren 1991, Vanbeveren et al. 1998; Eggleton 2002).

The energy formalism in its parameterized form is a convenient way to predict CE outcomes, but, as argued now, it is not necessarily a well-founded method (Ivanova et al. 2020). While at the moment, there is no complete understanding of the $\mathrm{CE}$ evolution in all the cases, it is understood that the $\mathrm{CE}$ event could be preceded by stable mass transfer (MT) on different timescales. The criteria for the occurrence of CE phase are still under development as well.

New stellar mass loss models (Ge et al.2010, 2015, 2020ba) and detailed simulations for close, mass exchanging binaries (Pavlovskii et al. 2017; Misra et al. 2020) have shown that RLOF may be stable over a much wider parameter space than previously thought. The same studies indicate that RLOF stability depends not only on the system mass ratio and the envelope type (convective or radiative), but also e.g., on the metallicity, stellar type or radius of the donor star. Those results begin to find confirmations when comparing theoretical models with the observed systems (Cherepashchuk et al. 2019; Leiner \& Geller 2021). The summary of the recent progress on RLOF stability has been summarized in $\$ 2.2$ of Klencki et al. (2021). Unfortunately, due to scarcity of observations for massive stellar systems during the ongoing RLOF phase and the high calculation costs, the current CE study (observations and simulations) usually refers to lowmass binary systems (Nandez et al. 2015, Nandez \& Ivanova
2016. Jones 2020). Such systems are not progenitors of BH-BH, BH-NS or NS-NS binaries.

CE phase is a key element setting the formation of DCOs in the classical isolated binary evolution channel and therefore understanding of $\mathrm{CE}$ is crucial in studies of origin of merging DCOs. Recently more and more signals form BH-BH, BH-NS and NS-NS mergers have been detected by LIGO/Virgo instruments (Abbott et al. 2019, The LIGO Scientific Collaboration et al. 2020) during the $\mathrm{O} 1, \mathrm{O} 2$ and $\mathrm{O} 3$ runs with reported parameters of systems such as the masses, spins and redshifts. It is still unknown what fraction of gravitational wave $(\mathrm{GW})$ signal mergers formed through isolated binary evolution in the field (Bond \& Carr 1984; Tutukov \& Yungelson 1993; Lipunov et al. 1997, Voss \& Tauris 2003; Belczynski et al. 2010, Dominik et al. 2012, Kinugawa et al. 2014; Mennekens \& Vanbeveren 2014 Hartwig et al. 2016, Spera et al. 2016; Belczynski et al. 2016b Eldridge \& Stanway 2016; Woosley 2016; Stevenson et al. 2017 Kruckow et al. 2018; Hainich et al. 2018, Marchant et al. 2019. Spera et al. 2019, Bavera et al. 2020), the dense stellar system dynamical channel (Miller \& Hamilton 2002b a; Portegies Zwart et al. 2004, Gültekin et al. 2004, 2006, O'Leary et al.| 2007 Sadowski et al. 2008; Downing et al. 2010, Antonini \& Perets 2012a; Benacquista \& Downing 2013; Bae et al. 2014; Chatterjee et al. 2017;: Mapelli 2016; Hurley et al.2016; Rodriguez et al. 2016, VanLandingham et al. 2016, Askar et al. 2017; Morawski et al. 2018, Banerjee 2018; Di Carlo et al. 2019; Zevin et al. 2019. Arca-Sedda \& Capuzzo-Dolcetta 2019; Rodriguez et al. 2018; Perna et al. 2019; Kremer et al.|2020); isolated multiple (triple, quadruple) systems (Antonini et al. 2017; Silsbee \& Tremaine 2017; Arca-Sedda et al. 2018; Liu \& Lai 2018 Fragione \& Kocsis 2019), mergers of binaries in galactic nuclei (Antonini \& Perets 2012b; Hamers et al. 2018, Hoang et al. 2018, Fragione et al. 2019); the chemically homogeneous evolution channel consisting of rapidly spinning stars in isolated binaries (de Mink \& Mandel 2016, Mandel \& de Mink 2016 Marchant et al. 2016, du Buisson et al. 2020) or Population III origin DCO binary mergers (Bond \& Carr 1984, Kinugawa et al. 2014, Tanikawa et al. 2021).

Over the years different groups developed their population synthesis codes and try to put better constrains on astrophysical processes by comparing theoretical model results with the known Galactic and extragalactic compact object population (e.g., Portegies Zwart \& Verbunt 1996, Hurley et al. 2002 Kruckow et al. 2018, Belczynski et al. 2020, Han et al. 2020). Several commonly encountered uncertainties in population synthesis studies strongly influence formation of DCOs and therefore, are subjects of active research. Examples of uncertain processes and parameters which are crucial for DCO mergers evolution are metallicity-specific star formation rate density (Chruślińska et al. 2020, Santoliquido et al. 2021;, Broekgaarden et al. 2021), NS and BH natal kicks (Mandel et al. 2021) or MT during stable/unstable RLOF (Vinciguerra et al. 2020; Howitt et al. 2020; Bavera et al. 2020).

In this paper, we study how applying the most recent developments in the understanding of RLOF stability into the population synthesis affects DCOs formation. In $\$ 2$ we describe the general method and the input physics implemented in the current version of StarTrack population synthesis code. In $\$ 3$ we introduce the revised CE development criteria and modified stable RLOF treatment examined in this paper. In $\S 4$ we present the results of our simulations: DCO local merger rate density, $\mathrm{BH}-\mathrm{BH}$ and $\mathrm{BH}-\mathrm{NS}$ mass ratio distributions and $\mathrm{BH}-\mathrm{BH}$ mass distributions for three tested models. $\S 5$ is a description of evolutionary scenarios leading to the $\mathrm{BH}-\mathrm{BH}$ and $\mathrm{BH}-\mathrm{NS}$ mergers formation 
in three tested models. It also includes three diagrams with an example of systems evolution. $\$ 6$ contains a brief discussion of our results together with the conclusions.

\section{Method}

To simulate formation and mergers of DCO systems in the local Universe we used updated StarTrack population synthesis code, developed over the years (Belczynski et al. 2002, 2008). The code allows to simulate evolution of a single star as well as binary star system for a wide range of initial conditions and physical parameters. Currently used version of implemented physics, adopted star formation history (SFH) and metallicity of the Universe is described in Belczynski et al. (2020) with two recent modifications, both explained in $\$ 2$ of Olejak et al. (2020). We adopted 3-broken power-law initial mass function (IMF) Kroupa et al. (1993); Kroupa (2002), weak pulsation pairinstability supernovae (PPSN) and pair-instability supernovae (PSN) (Woosley 2017, Belczynski et al. 2016a). We apply procedures for accretion onto a compact object during stable RLOF and from stellar winds based on the analytic approximations described in King et al. (2001) and Mondal et al. (2020). For non-degenerate accretors we adopt 50\% non-conservative RLOF (Meurs \& van den Heuvel 1989; Vinciguerra et al. 2020) with a fraction of the lost donor mass accreted onto the companion $\left(f_{a}=0.5\right)$, and the rest of mass $\left(1-f_{a}\right)$ leaving the system together with part of the donor and orbital angular momentum (see $\$ 3.4$ of Belczynski et al. 2008). We use 5\% Bondi-Hoyle rate accretion onto the compact object during the CE phase (Ricker \& Taam 2008; MacLeod \& Ramirez-Ruiz 2015; MacLeod et al. 2017). The procedure is based on equations 5.3-5.7 of (Bethe $\&$ Brown 1998) and equations A1-A10 of Belczynski et al.|(2002), and has been recently summarized in Appendix B of Olejak et al. (2020). For stellar winds we use formulas based on theoretical predictions of radiation driven mass loss (Vink et al. 2001) with inclusion of Luminous Blue Variable mass loss (Belczynski et al. 2010). All tested models have our standard physical values for the envelope ejection efficiency $\alpha_{\mathrm{CE}}=1.0$ and Maxwellian distribution natal kicks with $\sigma=265 \mathrm{~km} \mathrm{~s}^{-1}$ (Hobbs et al. 2005) lowered by fallback (Fryer et al. 2012). We adopt solar metallicity $\mathrm{Z}=0.02$ consistently with Pavlovskii et al. (2017).

We assume that systems with Hertzsprung gap (HG) donor star merge during CE phase (Belczynski et al. 2007). In StarTrack code HG phase begins after leaving the main sequence and for both less and more massive stars it is the period of intense star expansion (as during the main sequence stellar radius usually does not increase more than by a factor of few). Therefore during HG phase stars often initiate TTMT and CE. At the onset of RLOF such donors are often only partially expanded post main sequence stars. Therefore, it is not well known whether such objects have already well-separated core and envelope structure. In Figure 1 we present Hertzsprung-Russell evolution diagram for massive single stars. On the top panel of the figure we marked part of the evolution when stars are expected to have radiative or convective envelopes. On the bottom panel we marked part of evolution where stars are defined in StarTrack as main sequence+HG or core helium burning.

We tested delayed supernovae (SN) engine (Fryer et al. 2012; Belczynski et al. 2012) which affect the birth mass of NSs and BHs, allowing for the formation of the compact objects within the first mass gap $\left(\sim 2-5 M_{\odot}\right)$. We assume that maximum mass of the NS is $2.5 M_{\odot}$ (Horvath et al. 2020) so more massive compact objects are BHs.
Table 1. List of tested evolutionary models.

\begin{tabular}{lcc}
\hline Model & CE development criteria & MT switch \\
\hline \hline M380.B & Standard & Standard \\
M480.B & Revised & Standard \\
M481.B & Revised & Modified \\
\hline
\end{tabular}

In this work we present results for three different evolutionary models: a model with standard Startrack treatment of RLOF: M380.B and the other two which include the revised RLOF treatment: M480.B and M481.B. In the names "M" stands for a model, then the number is increasing with time to mark subsequent physical models. The ending "B" refers to submodel B often used in previous Startrack works e.g., Belczynski et al. (2020). In submodel B all HG donor systems merge during the CE phase. Models are listed in Table 1

\section{RLOF}

Two new types of instabilities were identified in RLOF binaries with massive donors, if such donors were allowed to evolve while they are exceeding their Roche lobe - the expansion and the convective instability (Pavlovskii et al.2017). Expansion instability happens if an RLOF donor experiences a period of fast thermal-timescale expansion after its main sequence. This expansion may lead to the development of dynamical instability in a very short time (a few thousand years) after the start of thermal time-scale mass transfer (TTMT). The second type of instability, convection instability, is associated with developing a sufficiently deep convective envelope.

In this work, we implement in our population synthesis studies the revised development criteria for the occurrence of the two instabilities and examine how they influence the formation of DCO mergers. We base our criteria for whether any of the instabilities occur in a given donor on the numerical results of Pavlovskii et al. (2017), who reported the boundaries between the instabilities using the radii of the donors of different masses and metallicities at the onset of RLOF. Following Pavlovskii et al. (2017) we define two radii: $R_{\mathrm{U}^{-}}$smallest radius for which the convection instability occurs, and $R_{\mathrm{S}}$ - the maximum radius when the expansion instability can take place.

In our approximation, the values of $R_{\mathrm{U}}$ and $R_{\mathrm{S}}$ are the averages of the ranges obtained and given in Table 1 of Pavlovskii et al. (2017). The specific values of $R_{\mathrm{U}}$ and $R_{\mathrm{S}}$ used in this work are given in Table 2 . Note that simulations by Pavlovskii et al. (2017) were performed for the systems with BH companion. In our work, we extend the results and treat the same way binary systems with any other companion types (e.g., main-sequence stars, neutron stars, etc.). If radius $R_{\text {don }}$ of a given donor at the onset of RLOF is found within range $R_{\mathrm{S}}-R_{\mathrm{U}}$ for $Z \leq 0.5 Z_{\odot}$ or below $R_{\text {don }}<R_{\mathrm{U}}$ for $Z>0.5 Z_{\odot}$ then we assume stable RLOF, otherwise CE evolution is applied. Note that stars of a higher metallicity were not found to experience the expansion instability (Pavlovskii et al.2017).

\subsection{CE development criteria}

In the revised treatment, we require the following four conditions to be met simultaneously for CE development:

(i) The condition based on the donor type. Revised RLOF instability criteria are applied if, during the RLOF, the donor is 

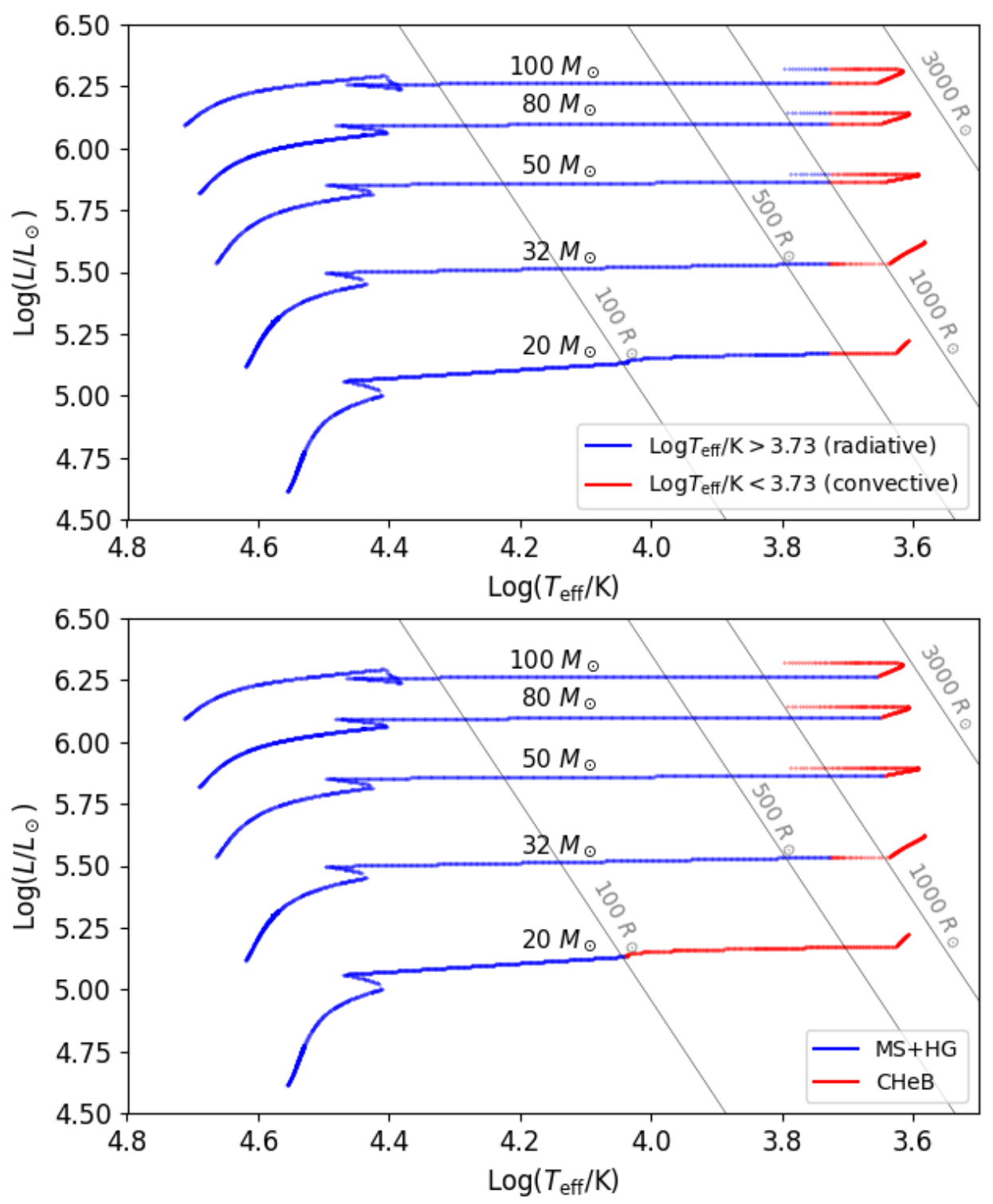

Fig. 1. Hertzsprung-Russell diagram for massive stars with ZAMS masses: $20 M_{\odot}, 32 M_{\odot}, 50 M_{\odot}, 80 M_{\odot}$ and $100 M_{\odot}$ at metallicity Z=0.007; evolution of single star since main sequence to core helium burning. In the top panel with the red line we marked stars cooler than $\log \left(T_{\text {eff }} / K\right)$ $<3.73$ and with the blue line stars with $\log \left(T_{\mathrm{eff}} / \mathrm{K}\right)>3.73$. It is expected that the red line represents stars having convective envelopes while the blue line is for radiative envelopes (Ivanova \& Taam 2004, Belczynski et al. 2008). In the bottom panel we marked with the blue line star main sequence and Hertzsprung gap phases and with the red line core helium burning phase. Evolutionary stages are defined as in formulas by Hurley et al. (2002) and Belczynski et al. (2008). With the grey lines we marked borders corresponding to stellar radii: $100 R_{\odot}, 500 R_{\odot}$ and $1000 R_{\odot}$.

an H-rich envelope giant of one of the following types: HG star, first giant branch, core helium burning, early asymptotic giant branch, or thermally pulsing asymptotic giant branch. However, for $\mathrm{HG}$ donors, we assume that $\mathrm{CE}$ always leads to a merger, and we halt this system binary evolution (see $\$ 2$ ).

(ii) The condition based on the donor mass. Revised RLOF instability criteria are applied only to the systems with initially massive donor stars with $M_{\mathrm{ZAMS}, \mathrm{don}}>18 \mathrm{M}_{\odot}$. (iii) The condition based on the system mass ratio. The ratio of companion masses $M_{\text {comp }}$ to donor masses $M_{\text {don }}$ (see Table 2) represents the border between regimes of always stable and possibly unstable RLOF. Therefore, from Table 2 we can obtain the limit value of mass ratio $q_{\mathrm{CE}}=M_{\text {comp }} / M_{\text {don }}$ for binary to enter potentially unstable RLOF regime. For metallicities $Z \leq$ $0.5 Z_{\odot}$ and for different donor mass $M_{\text {don }}$ ranges, $q_{\mathrm{CE}}$ values are 
Table 2. Boundary radii values between the stable and unstable RLOF, values based on data from Pavlovskii et al. (2017). If radius $R_{\text {don }}$ of a given donor during the ongoing RLOF is found within range $R_{\mathrm{S}}-R_{\mathrm{U}}$ for $Z \leq 0.5 Z_{\odot}$ or below $R_{\mathrm{don}}<R_{\mathrm{U}}$ for $Z>0.5 Z_{\odot}$ then we assume stable RLOF, otherwise CE evolution is applied. There are cases where expansion instability was not found for any radius (stable) and where cases where RLOF is unstable over whole radius range (unstable).

\begin{tabular}{cccc}
\hline$M_{\text {don }}\left[M_{\odot}\right]$ & $M_{\text {comp }}\left[M_{\odot}\right]$ & $R_{\mathrm{S}}\left[R_{\odot}\right]$ & $R_{\mathrm{U}}\left[R_{\odot}\right]$ \\
\hline \hline \multicolumn{4}{c}{ Metallicity $Z \leq 0.5 Z_{\odot}$} \\
\hline 19.6 & 7 & stable & 703.5 \\
29.1 & 7 & 47.5 & 1057.5 \\
37.6 & 7 & 331.5 & 1293.5 \\
56.8 & 7 & \multicolumn{2}{c}{ unstable } \\
56.8 & 10 & 355.0 & 1747.5 \\
56.8 & 12 & 148.0 & 1823.5 \\
74.5 & 7 & \multicolumn{3}{c}{ unstable } \\
74.5 & 10 & stable & 2229.0 \\
74.5 & 14 & 144.5 & 2150.5 \\
\hline \multicolumn{5}{c}{} \\
\hline 19.6 & 7 & stable & 736.0 \\
26.6 & 7 & stable & 1159.0 \\
32.5 & 7 & stable & 1407.5 \\
41 & 10 & stable & 2103.5 \\
41 & 12 & stable & 2033.0 \\
\hline \multicolumn{5}{c}{}
\end{tabular}

following:

$\begin{array}{llll}q_{\mathrm{CE}}=0.36 & \text { for } & M_{\mathrm{don}} \in(18,60) M_{\odot} \\ q_{\mathrm{CE}}=0.21 & \text { for } & M_{\mathrm{don}} \in[60,80) M_{\odot} \\ q_{\mathrm{CE}}=0.19 & \text { for } & M_{\mathrm{don}} \geq 80 M_{\odot}\end{array}$

while for metallicities $Z>0.5 Z_{\odot}$

$$
\begin{array}{llrl}
q_{\mathrm{CE}}=0.36 & \text { for } & M_{\mathrm{don}} \in(18,60) M_{\odot} \\
q_{\mathrm{CE}}=0.29 & \text { for } & M_{\mathrm{don}} \in[60,80) M_{\odot} \\
q_{\mathrm{CE}}=0.19 & \text { for } & & M_{\mathrm{don}} \geq 80 M_{\odot}
\end{array}
$$

If the mass ratio of the binary system is greater than $q_{\mathrm{CE}}$, we assume stable RLOF.

(iv) The condition based on the donor radius. Finally, if all conditions i-iii are met, we use stability diagrams presented in Figures 2 and 3 to decide between stable RLOF and CE development. Diagrams are for two metallicities corresponding to simulations performed by Pavlovskii et al. (2017): $Z=0.1 Z_{\odot}$ and $Z=1.0 Z_{\odot}$ respectively. We marked the values of $R_{\mathrm{U}}$ and $R_{\mathrm{S}}$ with the corresponding donor masses taken from the Table 2 and fit functions to the simulation points. We extend our model for wider metallicity ranges and follow different procedures for metallicities smaller than $0.5 Z_{\odot}$ and greater than $0.5 Z_{\odot}$.

For $Z \leq 0.5 Z_{\odot}$ we used simulation data for $Z=0.1 Z_{\odot}$ and we fit second-degree polynomial to $R_{\mathrm{S}}$ points with the following coefficients:

$R_{\mathrm{S}}=-0.29 M_{\mathrm{don}}^{2}+30.3 M_{\mathrm{don}}-498$

and a straight line to $R_{\mathrm{U}}$ :

$R_{\mathrm{U}}=26.3 M_{\mathrm{don}}+262$.

If a donor during ongoing RLOF has a radius $R_{\mathrm{don}}<R_{\mathrm{S}}$ or $R_{\mathrm{don}}$ $>R_{\mathrm{U}}$, RLOF is unstable $(\mathrm{CE})$.
For $Z>0.5 Z_{\odot}$ we used simulation data for $Z=1.0 Z_{\odot}$. In this case, we had only $R_{\mathrm{U}}$ points and we fit a straight line with the equation:

$R_{\mathrm{U}}=62.3 M_{\mathrm{don}}-515$.

If a donor radius during ongoing RLOF is $R_{\mathrm{don}}>R_{\mathrm{U}}$, RLOF is unstable (CE).

$M_{\text {don }}$ in Equations 46 stands for the mass of the donor at a given RLOF time step, such that only $1 \%$ of donor mass is transferred (see §5.1, Belczynski et al. (2008)).

In detailed simulations, RLOF is always unstable if $q<$ 0.123 (see also Table 2). We therefore adopt that CE event always starts in binaries where at the onset of RLOF the mass ratio is

$\frac{M_{\text {comp }}}{M_{\text {don }}}<q_{\text {crit }}=0.125$.

Unfortunately, He giant donors do not have envelopes similar to hydrogen stars to make a straightforward connection and use revised RLOF stability diagrams. Therefore, in this work for He stars as well as in cases when the donor is a main sequence star (H-rich; core H-burning and He-rich; core He-burning main sequence) or a giant of $M_{\mathrm{ZAMS} \text {,don }}<18 M_{\odot}$ we follow the standard StarTrack CE development criteria and stable RLOF treatment (Belczynski et al.2008).

\subsection{Stable RLOF}

In this study, we also test a modification of the condition for switching from TTMT to nuclear-timescale stable MT in the StarTrack code. So far, once the TTMT began, the issued MT was continued until the donor star's radius $R_{\text {don }}$ decreased below 1.1 of its Roche lobe radius $R_{\text {lobe }}$. Then the type of MT was selected based on the comparison of MT timescale $\tau_{\text {eq }}$ (Equation 46 from Belczynski et al. 2008) and thermal timescale $\tau_{\text {thermal }}$. For $\tau_{\text {thermal }}$ we use the approximation from Kalogera \& Webbink (1996):

$\tau_{\text {thermal }} \approx 30\left(\frac{M_{\mathrm{don}}}{M_{\odot}}\right)^{2}\left(\frac{R_{\mathrm{don}}}{R_{\odot}}\right)^{-1}\left(\frac{L_{\mathrm{don}}}{L_{\odot}}\right)^{-1} \mathrm{Myr}$.

If $\tau_{\text {eq }}>\tau_{\text {thermal }}$, it was assumed that MT is driven on nuclear timescale. If $\tau_{\text {eq }} \leq \tau_{\text {thermal }}$, it was adopted the MT proceeds on thermal timescale.

In one of the tested models of this work, we modified this condition allowing for the switch from TTMT to nuclear timescale stable MT always based on the timescale comparison in the given RLOF time step (not only if $R_{\text {don }}<1.1 R_{\text {lobe }}$ ). Note that this modification is applied to all binaries during their RLOF, regardless of components masses or types (as opposed to the new CE development criteria described in the $\$ 3.1$.

The evolutionary formulas we employ to evaluate star properties (e.g., radii, mass, luminosity) based on Hurley et al. (2000) are not applicable for thermal-timescale changes of the star outside of thermal-timescale evolution that is intrinsic to normal single stars (e.g., post-main-sequence expansion). Therefore, we can not properly evaluate the donor's properties during ongoing TTMT, as then the donor is out of its thermal equilibrium. We obtain TTMT rate using mass, radius, and luminosity that the donor had when the TTMT just had started. Hence, the donor is still in thermal equilibrium. Therefore, we assume that the donor's properties should still be approximated well by our parameterized equations. This obtained TTMT rate is kept constant 
for as long as TTMT proceeds. We have to resort to this simplified way to obtain MT rate as, while TTMT proceeds, our evolutionary formulas generate unreliable star radius and luminosity.

Yet, we use the onset RLOF parameters to construct our switches. In the first (original StarTrack) switch from TTMT to nuclear timescale MT, we wait until the donor's radius, as given by the evolutionary formula, becomes comparable to the size of the donor's Roche lobe. Then we assume that the star is back (or close) to its thermal equilibrium. Then we use the new donor's properties to decide whether to continue TTMT (with a new MT rate based on the current donor mass, radius, and luminosity), or change to nuclear timescale MT.

In the modified approach that we test in this work, at each time step of the ongoing TTMT we use the evolutionary formulae and calculate the current thermal and nuclear timescales. Suppose we get that the updated nuclear timescale is longer than the updated thermal timescale and the donor's radius is still larger than its Roche lobe radius. In that case, we assume that the donor has regained its thermal equilibrium, and we proceed with the MT on the new nuclear timescale. This usually happens earlier in the ongoing TTMT than meeting the original condition $R_{\text {don }}<1.1 R_{\text {lobe }}$. With this new additional condition, a numerical artifact is plausible: the donor star's radius may significantly exceed its Roche lobe when we change MT to nuclear timescale. In this case we apply a "safety" condition such that if

$R_{\text {don }}>2.0 R_{\text {lobe }}$

we proceed with $\mathrm{CE}$ evolution for giant donors, or assume merger for main sequence donors (both $\mathrm{H}$-core burning $\mathrm{H}$-rich and He-core burning He-rich main sequence stars).

Using either the original switch or the modified one is not perfect. Since we cannot be sure which transition condition from TTMT to nuclear timescale MT would correspond better to detailed MT calculations, we will present models with both treatments.

\subsection{Other cases of unstable RLOF}

There are other situations when RLOF is unstable, leading to a CE phase. We take into account the following two of the known scenarios: $(i)$ in case of the Darwin instability, due to an extreme mass ratio (Lai et al. 1993); (ii) if the accretion flow is so strong that photons are trapped in it, building up an envelope around a compact accretor in excess of its Roche lobe radius (Begelman|1979). For these, we use standard StarTrack procedures (Belczynski et al.|2008).

\section{Results}

\subsection{NS-NS, BH-NS and $B H-B H$ local merger rate density}

We present local merger rate densities $(z \sim 0)$ for different types of DCO systems corresponding to the tested physical models with standard (M380.B) and revised RLOF treatment (M480.B, M481.B) listed in the Table 3. Our rates are placed together with values recently estimated by LIGO/Virgo (90\% credible limits): $23.9_{-8.6}^{+14.9} \mathrm{Gpc}^{-3} \mathrm{yr}^{-1}$ for BH-BH mergers, $320_{-240}^{+490}$ $\mathrm{Gpc}^{-3} \mathrm{yr}^{-1}$ for NS-NS mergers (The LIGO Scientific Collaboration et al. 2020) and previously given range for BH-NS mergers: $0-610 \mathrm{Gpc}^{-3} \mathrm{yr}^{-1}$ (Abbott et al. 2019).

The effect of revised CE development criteria (separate from other tested changes) on the merger rates is visible by comparing results of model M380.B and M480.B. It seems to be rather counterintuitive as the rate for $\mathrm{BH}-\mathrm{BH}$ and $\mathrm{BH}-\mathrm{NS}$ mergers increased under more restricted conditions for $\mathrm{CE}$ initiation. In standard model (M380.B) the local merger rate densities were for BH-BH: $62 \mathrm{Gpc}^{-3} \mathrm{yr}^{-1}$ and for BH-NS: $13 \mathrm{Gpc}^{-3} \mathrm{yr}^{-1}$ while in M480.B they slightly increased to $88 \mathrm{Gpc}^{-3} \mathrm{yr}^{-1}$ and $16 \mathrm{Gpc}^{-3} \mathrm{yr}^{-1}$ respectively. This is caused by the fact that in the revised treatment during the RLOF some of the systems with HG star donor instead of entering the unstable RLOF regime (which always lead to the merger, $\$ 2$, go through the period of TTMT which allows for further binary evolution and potential formation of BH-BH systems. On the other hand, systems initiate $\mathrm{CE}$ phase much less often and as the consequence we obtain new, dominant evolutionary scenario leading to formation of $\mathrm{BH}-\mathrm{BH}$ mergers without any CE phase. In other words, the early (HG donors) development of CE during TTMT eliminates many progenitors of BH-BH systems from dominant formation channel of model M480.B and the restricted (in terms of mass and mass ratio) development of $\mathrm{CE}$ reduces number of $\mathrm{BH}-\mathrm{BH}$ mergers from the dominant formation channel in model M380.B. For details about the new evolutionary scenario see $\$ 5$. The rate for NS-NS mergers (model M480.B), $\sim 150 \mathrm{Gpc}^{-3} \mathrm{yr}^{-1}$, is the same as in the standard model (M380.B), as the revised CE development criteria were adopted only to the systems with initially massive donors $\left(M_{\text {ZAMS }}>18 M_{\odot}\right.$, see $\$ 3.1$ which are mainly $\mathrm{BH}$ progenitors. Therefore NS-NS systems formation was not significantly influenced and the merger rate is within LIGO/Virgo range as it used to be for the standard approach. Rates for BH-NS systems for both M380.B and M480.B models are also consistent with the given LIGO/Virgo range, which is wide due to the limited detection data for BH-NS mergers (The LIGO Scientific Collaboration et al. 2020). BH-BH merger rates for the same two models are about 1.5-2.0 times larger than the upper limit given by LIGO/Virgo.

Model M481.B includes both implemented changes to our standard approach (M380.B): revised CE development criteria and modified condition for switch between TTMT and nuclear timescale stable MT. As the second change was implemented to all systems (regardless components masses or types) it affected also NS-NS merger rate (for M481.B equal $320 \mathrm{Gpc}^{-3} \mathrm{yr}^{-1}$ ) increasing it about 2 times comparing with the M380.B and M480.B models. The additional modification implemented in model M481.B leads, however, to decrease of the rate for $\mathrm{BH}-$ $\mathrm{BH}$ and BH-NS mergers (18 $\mathrm{Gpc}^{-3} \mathrm{yr}^{-1}$ and $4 \mathrm{Gpc}^{-3} \mathrm{yr}^{-1}$ respectively) by a factor of $\sim 4-5$ comparing to the model M480.B. The effect described in previous paragraph which leads to the increase of the survived $\mathrm{BH}$ binaries progenitors number due to entering stable RLOF instead of early CE with HG donors, does not apply here. Due to the modification of condition for switch between MT types, TTMT stops faster than in the standard approach. At the same time donor's radius derived from the formula for stars in equilibrium (see $\$ 3.2$ ) is large $\left(R_{\text {don }}>2.0 R_{\text {lobe }}\right)$ what leads to early CE initiation (and merger of all $\mathrm{HG}$ donor systems). The new dominant $\mathrm{BH}-\mathrm{BH}$ merger formation channel emerges in this model and it is different than dominant BH-BH formation channels in models M380.B and M480.B. This channel includes TTMT that develops into late CE with core helium burning donor (for details see \$5). Merger rates for all types of DCOs in this (M481.B) model are in good agreement with recent LIGO/Virgo estimates.

\subsection{BH-NS and BH-BH mass ratio distribution}

Until the realise of $\mathrm{O} 3$ data, all ten detected $\mathrm{O} 1 / \mathrm{O} 2 \mathrm{BH}-\mathrm{BH}$ mergers published by LIGO/Virgo team were consistent with be- 
Table 3. Local $(z \sim 0)$ merger rate densities $\left[\mathrm{Gpc}^{-3} \mathrm{yr}^{-1}\right]$ for different types of DCO systems. With the bold font we mark the model for which $\mathrm{BH}-\mathrm{BH}, \mathrm{BH}-\mathrm{NS}$ and NS-NS merger rates are within ranges given by LIGO/Virgo (Abbott et al.2019, The LIGO Scientific Collaboration et al. 2020).

\begin{tabular}{lccc}
\hline Model & $\mathcal{R}_{\mathrm{BH}-\mathrm{BH}}$ & $\mathcal{R}_{\mathrm{BH}-\mathrm{NS}}$ & $\mathcal{R}_{\mathrm{NS}-\mathrm{NS}}$ \\
\hline \hline LIGO/Virgo & 23.9 & - & 320 \\
& $15.3-38.8$ & $0-610$ & $80-810$ \\
\hline M481.B & $\mathbf{1 7 . 9}$ & $\mathbf{4 . 1}$ & $\mathbf{3 2 2}$ \\
M380.B & 61.7 & 13.1 & 148 \\
M480.B & 88.4 & 15.6 & 148 \\
\hline
\end{tabular}

ing equal-mass merges (Abbott et al. 2019a b; Fishbach \& Holz 2020). Latest detections indicated however, that the distribution of $\mathrm{BH}-\mathrm{BH}$ mergers mass ratio may be more complex and broad. Recently two detections of DCO mergers with highly asymmetric masses were announced (The LIGO Scientific Collaboration \& the Virgo Collaboration 2020, Abbott et al.2020a). The events GW190412 $\left(\sim 30 M_{\odot}\right.$ BH and $\sim 8 M_{\odot}$ BH $)$ and GW190814 $\left(\sim 23 M_{\odot} \mathrm{BH}\right.$ and $\sim 2.6 M_{\odot} \mathrm{NS}$ or $\left.\mathrm{BH}\right)$ were caused by DCO mergers in which one of the objects was less massive than the second by a factor of $0.28_{-0.07}^{+0.12}$ and $0.112_{-0.009}^{+0.008}$ respectively. We check how revised CE development criteria and stable RLOF treatment influence the mass ratio distribution of $\mathrm{BH}-\mathrm{BH}$, and BH-NS mergers, and if we are still able to reconstruct asymmetric mass $\mathrm{BH}-\mathrm{BH}$ and $\mathrm{BH}-\mathrm{NS}$ mergers as in previous models (Olejak et al. 2020, Drozda et al. 2020). In our study, we define mass ratio of merging components $q$ as the ratio of less massive compact object to the more massive compact object in agreement with LIGO/Virgo definition.

In Figures 4 and 5 we present distributions of mass ratio of $\mathrm{BH}-\mathrm{BH}$ and $\mathrm{BH}-\mathrm{NS}$ mergers $(z \sim 0)$ for two models with revised RLOF treatment (red line): M480.B and M481.B respectively.

We calculated percent of asymmetric mass BH-BH mergers with $q<0.4$ which has been constrained after GW190412 detection to constitute $\gtrsim 10 \%$ of overall $\mathrm{BH}-\mathrm{BH}$ merger population (The LIGO Scientific Collaboration \& the Virgo Collaboration 2020). In the standard StarTrack models with rapid (M230.B, Olejak et al. (2020)) and delayed (M380.B) SN engine (Fryer et al. (2012)) those fractions are $9 \%$ and $\sim 30 \%$ respectively. We find that our tested revised $\mathrm{CE}$ development criteria reduced the fraction of unequal $\mathrm{BH}-\mathrm{BH}$ mergers comparing to model M380.B. Percent of BH-BH mergers with $q<0.4$ for model M480.B constitute $\sim 2 \%$ while for M481.B it is $\sim 6 \%$. The main formation channel for the formation of unequal mass BH-BH binaries in standard approach (see Tab.1 of Olejak et al. (2020)) is missing for the revised CE treatment (see Tab.4, §5). Systems that would produce unequal mass $\mathrm{BH}-\mathrm{BH}$ mergers, in the revised treatment instead of $\mathrm{CE}$, enter stable RLOF regime and finally form wide $\mathrm{BH}-\mathrm{BH}$ binaries which do not merge in Hubble time. The lowest $\mathrm{BH}-\mathrm{BH}$ merger mass ratio achieved in tested models is $q \sim 0.06$ for the M480.B and $q \sim 0.1$ for M481.B.

In distribution of $\mathrm{BH}-\mathrm{BH}$ mergers for model M480.B there is a large peak in the range of $q \approx 0.4-0.6$ which is not present in distributions for other models. Vast majority (94\%) of BH-BH mergers in M480.B forms via evolutionary scenario without any CE phase (see Tab. 4 and Fig. 9). Binary separation, instead during $C E$ phase as in the standard Startrack approach, is reduced as the donor mass is ejected from the system together with the or- bital angular momentum during stable RLOF. Described mechanism of orbital shrinkage is effective only for unequal mass binary components, when donor mass at the RLOF onset is significantly larger than the companion's mass (van den Heuvel et al. 2017, Marchant et al. 2021). In our simulations such systems usually end their evolution as $\mathrm{BH}-\mathrm{BH}$ binaries with mass ratio close to $q=0.5$ (Fig.9).

Mass ratio distribution for BH-NS mergers is strongly dominated with low mass ratio systems, what has been already reported and described for example by Drozda et al. (2020). For both models M480.B and M481.B, 90\% of the BH-NS mergers have $q<0.35$. However, the lowest achieved mass ratios for BH-NS merger $(\sim 0.1)$ is not that extreme as it used to be in standard model M380.B (0.03). Similarly as in case of the BH-BH system, the main formation channel leading to formation of extreme mass ratios (see Fig.6 in Drozda et al. (2020)) is reduced as during the first RLOF instead of CE systems goes through period of stable RLOF.

\section{3. $\mathrm{BH}-\mathrm{BH}$ mass distributions}

The LIGO Scientific Collaboration et al. (2020) gives recent estimations on the possible shape of mass distribution for the more massive of the merging BHs $\left(m_{1}\right)$ based on so far detected BH-BH mergers. The proposed fit is a power-law mass function $p\left(m_{1}\right) \propto m_{1}^{-\alpha}$ with a break around $40 M_{\odot}$ with the first exponent for the masses $m_{1} \lesssim 40 M_{\odot}, \alpha_{1}=1.58_{-0.86}^{+0.82}$ and the second exponent for $m_{1} \gtrsim 40 M_{\odot}, \alpha_{2}=5.6_{-2.5}^{+4.1}$.

We present the distributions of more massive, less massive $\mathrm{BH}$ masses and total mass of $\mathrm{BH}-\mathrm{BH}$ systems which merged at redshifts $z<2$ for two tested models with revised RLOF physics. The Figures 6 and 7 shows results for model M480.B and M481.B respectively. We approximate more $\mathrm{BH}$ mass distributions (Figs. 6 and 77 top panels) with power-law function for an easy visual comparison with the LIGO/Virgo estimate.

The shape of more massive BH distribution for our model M480.B is in a very good agreement with the shape of the $\mathrm{BH}$ BH detections (Fig. 6, top panel). The distribution for this model may be divided as well into two parts, with the break around $45 M_{\odot}$. The first part, up to the break is characterized by a slow decrease corresponding to the exponent $\alpha_{1} \approx 1.5$ while after the break, the curve become much steeper with $\alpha_{2} \approx 5.3$ and the cut at $\sim 55 M_{\odot}$ (PSN, begining of the second mass gap). Note that this model distribution is a better match to the LIGO/Virgo estimate than recently published StarTrack results (see Fig.15 for model M30.B in Abbott et al. (2020b): single power-law with index $\alpha=3.6$. The LIGO/Virgo total BH-BH system masses are within range of our simulated population of $\mathrm{BH}-\mathrm{BH}$ mergers for model M480.B. The only exception is GW190521: the most massive detected BH-BH merger (Abbott et al. 2020b), that has both BHs $\left(85 M_{\odot}+66 M_{\odot}\right)$ within our adopted pair-instability mass gap: 55 - $135 M_{\odot}$ (Belczynski et al. 2020). However, even such massive BHs can be possibly produced by massive stars if uncertainties on nuclear reaction rates and mixing in stellar interiors are taken into account (Belczynski 2020).

In the case of model M481.B, the more massive BH mass distribution is not a good match to LIGO/Virgo data. Although two power-law exponents $\left(\alpha_{1} \approx 1.0\right.$ and $\left.\alpha_{2} \approx 5.0\right)$ are within LIGO/Virgo estimates, the break appears at quite a low mass $M_{\text {break }} \approx 15-20 M_{\odot}$. Note also the total BH-BH mass distribution ends at lower mass $\left(M_{\text {cutoff }}=100 M_{\odot}\right)$ as compared with model M480.B $\left(M_{\text {cutoff }}=110 M_{\odot}\right)$. 
We also plot mass distributions for the model M380.B which represents our standard RLOF physics (Fig. 8). Similarly to M481.B, the break point of more massive BH mass distribution is at rather low BH masses, $M_{\text {break }} \approx 15-20 M_{\odot}$. However, in this case for masses $m_{1}<M_{\text {break }}$ the distribution is increasing with $\mathrm{BH}$ mass $\left(\propto M^{+2.7}\right)$, which is inconsistent with the LIGO/Virgo estimates. For masses $m_{1}>M_{\text {break }}$ we fit power-law exponent $\alpha_{2}=3.3$. The maximum total mass $\left(m_{1}+m_{2}\right)$ of the BH-BH merger for model M380.B is $110 M_{\odot}$.

Our results indicate that modifications in RLOF physics approach may drastically influence the $\mathrm{BH}$ mass distribution of BH-BH mergers. Some approaches (M480.B) allow to match nicely the empirical data for more massive $\mathrm{BH}$ mass distribution while for others distribution shape is rather off (M380.B and M481.B).

\section{Formation scenarios}

\subsection{BH-BH formation scenarios}

In Table 4 we provide evolutionary scenarios which lead to the formation of local $(z \sim 0) \mathrm{BH}-\mathrm{BH}$ mergers for tested models with revised CE development criteria (M480.B and M481.B) and standard StarTrack criteria (M380.B). For each model the dominant formation scenario, which constitutes the highest merger rate density fraction (marked with the bold text) is different. In Figures 9 and 10 we present diagrams with the example for the dominant BH-BH formation scenario in model M480.B (without $\mathrm{CE}$ phase) together with alternative evolution of the same system (the same initial conditions) but in models M380.B and M481.B. The Figure 9 is an example of a low-mass BH-BH merger formation, for which significant natal kick (received after second SN explosion) plays an important role. The second example (Fig. 10) is massive BH-BH merger formation in which two BHs form via direct collapse.

For model M480.B (revised CE development criteria and standard switch from TTMT to nuclear-timescale stable MT) the dominant BH-BH mergers formation scenario is without any CE phase. Similar channels were reported in many other recent works (van den Heuvel et al. 2017; Andrews et al. 2020; Zevin et al. 2020; Bavera et al. 2020; Marchant et al. 2021) as a possible formation scenario of LIGO/Virgo sources via isolated binary evolution. The typical evolution of binary system in model M480.B is following: when primary (initially more massive star) leaves its main sequence and expands, system goes through a period of TTMT. Note that in some cases the donor star enters TTMT while is at its HG. Survival of a binary through this phase is facilitated by high TTMT rate from the donor star. This removes most of (or entire) donor's H-rich envelope and disallow significant increase of donor radius which in turn does not allow for development of CE phase and possible merger of donor with its companion. After RLOF primary becomes a naked helium star. When primary star finishes evolution, the first BH is formed. In the meantime secondary evolves off main sequence, expands and initiates a second phase of TTMT. The second RLOF, as well as the first one, is often initiated by a HG stars expanding on the thermal scales. If the system components have highly unequal masses (the donor mass is by a factor of 3 or more greater than the $\mathrm{BH}$ mass) the large mass loss from the system carries away also lots of the orbital angular momentum (even 90\% comparing with the amount at the second RLOF onset). This leads to significant orbit tightening. Such a mechanism has been reported previously e.g., by van den Heuvel et al. (2017); Marchant et al.
(2021). After MT, the secondary becomes naked helium star, and next forms a second, typically less massive $\mathrm{BH}$.

In all tested models BHs with masses $M_{\mathrm{BH}}<10-15 M_{\odot}$ may receive a natal kick at the time of their formation (see $\$ 2$. The lower the $\mathrm{BH}$ mass, the greater probability of a high natal kick. Therefore, in the $\mathrm{BH}-\mathrm{BH}$ formation scenario the second-born $\mathrm{BH}$ (usually the less massive one) may receive a significant kick. High natal kick may impart significant eccentricity to newly formed $\mathrm{BH}-\mathrm{BH}$ system what decreases the inspiral timescale $T_{\text {ins }} \sim\left(1-e^{2}\right)^{7 / 2},($ Peters 1964), allowing such BH-BH system to merge in Hubble time. For example BH-BH system of $25 M_{\odot}$ and $10 M_{\odot}$ on the wide orbit of $\sim 900 R_{\odot}$ may merge in $\sim 10$ Gyr if the eccentricity is as high as $e=0.992$. For a comparison, in the case of circular orbit $(e=0)$ initial separation of the same $\mathrm{BH}-\mathrm{BH}$ system would have to be less than $\sim 30 R_{\odot}$, for eccentricity $e=0.7$ less than $\sim 45 R_{\odot}$ and for $e=0.9$ less than $\sim 110 R_{\odot}$. First stable RLOF phase in this scenario (M480.B) would be replaced in our standard model (M380.B) with CE phase for many binaries as revised CE development criteria is more restrictive. Obviously, CE may lead in some cases to binary component merger and formation of a massive single stars. Note however, that even in our standard scenario dominant formation channel, the first RLOF is stable TTMT from HG star (compare dominant path 3 for model M380.B with dominant path 1 for model M480.B in Tab. 4). The second stable RLOF phase would be replaced by CE in the standard model. The CE during second RLOF is the main phase which leads the orbit size decrease for final BH-BH system to merge within Hubble time (see path 3 in Tab. 4 for model M380.B).

For Model M481.B (revised CE development criteria and modified condition for switch from TTMT to nuclear timescale MT) the main BH-BH formation channel also differs from the one in the standard Startrack approach (M380.B). Most of the evolution resembles the scenario described for M480.B: first TTMT initiated by primary, then the first $\mathrm{BH}$ formation with possible natal kick and the second TTMT initiated by secondary. However, in model M481.B due to modified condition for switch from the TTMT to nuclear-timescale stable MT, and the "safety" condition (read $\S 3.2$ ), when TTMT ends system goes through $\mathrm{CE}$ phase. If the system survives $\mathrm{CE}$, the separation is reduced and the secondary's envelope is ejected leaving a naked helium core. Soon thereafter CE (in some cases also already during CE), the secondary's core collapse and the second (typically less massive) $\mathrm{BH}$ is formed.

\subsection{BH-NS formation scenarios}

In Table 5 we provide formation scenarios of local $(z \approx 0)$ BH-NS mergers for tested models with revised CE development criteria (M480.B and M481.B) and standard Startrack criteria (M380.B). For each model the dominant formation scenario which constitutes the highest merger rate density fraction is marked with the bold text. For two models M380.B and M480.B the dominant formation scenario is the same, however, for model M480.B it constitutes over $70 \%$ while for M380.B about $50 \%$ of total BH-NS merger rates. The evolutionary diagrams with the dominant formation scenarios are shown in Figure 11.

Most of the evolutionary phases are common for all three tested models. Evolution scenario goes as follows: once primary, initially more massive star, leaves main sequence, it expands and initiates first stable RLOF. System goes through the stable MT, after which the donor (primary) star looses its envelope becoming a stripped helium star. Next, primary forms a BH through $\mathrm{SN}$ explosion or via direct collapse (depends on the final star 
Table 4. Local $(z \sim 0)$ merger rate densities $\left[\mathrm{Gpc}^{-3} \mathrm{yr}^{-1}\right]$ for different $\mathrm{BH}-\mathrm{BH}$ formation scenarios. The main formation scenario for given model is marked with the bold text.

\begin{tabular}{llccc}
\hline No. & BH-BH formation scenario & $\mathcal{R}_{\mathrm{M} 480 . \mathrm{B}}$ & $\mathcal{R}_{\mathrm{M} 481 . \mathrm{B}}$ & $\mathcal{R}_{\mathrm{M} 380 . \mathrm{B}}$ \\
\hline \hline 1. & MT1(1/2/4/5-1/4)/MT2(7-4) BH1 MT2(14-2/4/5/7/8) BH2 & $\mathbf{8 3 . 1}$ & 0.4 & 0.3 \\
2. & MT1(1/2/4-1) BH1 MT2(14-2/4) SW CE2(14-4/5:14-7/8/14) BH2 & 0.4 & $\mathbf{1 2 . 5}$ & 0.0 \\
3. & MT1(1/2/4-1) BH1 CE2(14-4/5:14-7/8/14) BH2 & 2.1 & 2.0 & $\mathbf{5 1 . 2}$ \\
4. & MT1(1/2/4-1) BH1 MT2(14-2/4) CE2(14-4/5:14-7/8/14) BH2 & 1.1 & 1.0 & 1.6 \\
5. & MT1(4-2/4) MT2(4/5/7/8-4) CE12(4/5/8-4:14-7) BH1 BH2 & 1.4 & 1.4 & 2.0 \\
6. & Other scenarios & 0.3 & 0.6 & 6.6 \\
& Total & 88.4 & 17.9 & 61.7 \\
\hline
\end{tabular}

MT1-stable RLOF, donor is initially more massive star;

MT2-stable RLOF, donor is initially less massive star;

BH1-formation of black hole by initially more massive star;

$\mathrm{BH} 2$-formation of black hole by initially less massive star;

CE1-common envelope initiated by initially more massive star;

CE2-common envelope initiated by initially less massive star;

CE12-double common envelope initiated by two giants;

SW - switch from TTMT to CE based on the revised condition (see $\$ 3.2$ and $§ 5$ ).

The numeric types are consistent with Hurley et al. (2002):

0 - main sequence star with $\mathrm{M}<=0.7$ Msun (deeply or fully convective)

1 - main sequence star with $\mathrm{M}>0.7$ Msun

2 - Hertzsprung gap star

3 - first giant branch star

4 - core helium burning star

5 - early asymptotic giant branch star

6 - thermally pulsing asymptotic giant branch star

7 - main sequence naked helium star

8 - Hertzsprung gap naked helium star

9 - giant branch naked helium star

10 - helium white dwarf

11 - carbon or oxygen white dwarf

12 - oxygen or neon white dwarf

13 - neutron star

14 - black hole

15 - massless remnant

The values in the brackets, e.g., 1/2/4 refer to different possible variants of the evolutionary types of binary components during given phase. The type of initially more massive component is given as the first and the type of initially less massive component as a second (after dash,-). In the case of CE phase type at the onset are given as the first, next types after envelope ejection (after colon).

mass). When the secondary star leaves its main sequence it expands and initiates unstable MT. The CE development leads to the secondary's envelope removal and significant contraction of the system separation. Close system of BH and naked helium star is formed. As after CE phase the secondary is a main sequence or HG helium star, it still expands initiating a stable RLOF. At the end of the TTMT, the evolutionary scenarios for different models begin to diverge. In the case of models M380.B and M480.B, after TTMT system is close BH-He binary. Next, there is a SN explosion and the NS formation. In the scenario M481.B, due to modified condition for the switch between the TTMT and nuclear-timescale stable MT, and the "safety" condition (read $\$(3.2)$, the system ends stable RLOF with CE phase. The orbit is tightened once again. During the second CE phase the NS is formed.

\section{Conclusions and discussion}

In this work, we implement into StarTrack population synthesis code the revised RLOF stability diagram for binary systems with initially massive donors $\left(M_{\mathrm{ZAMS}}>18 M_{\odot}\right)$. The revised diagram is based on MT simulations performed by Pavlovskii et al. (2017) using 1D hydrodynamical stellar code MESA. They found that RLOF is stable over a much wider parameter space than previously thought if the MT is allowed to proceed until outflows from the outer Langrangian points develop. For one tested model, we also modify conditions for switching between TTMT and nuclear timescale MT. We used the most updated version of StarTrack code to calculate local merger rate density and mass ratio distribution of the synthetic population of DCOs mergers.

We compare the results of the revised RLOF treatment with the standard StarTrack. We also check if new results are consistent with the recent estimates of LIGO/Virgo collaboration, comparing local merger rate density, the fraction of unequal 
Table 5. Local $(z \sim 0)$ merger rate densities $\left[\mathrm{Gpc}^{-3} \mathrm{yr}^{-1}\right]$ for different $\mathrm{BH}-\mathrm{NS}$ formation scenarios. The main formation scenario for given model is marked with the bold text.

\begin{tabular}{llccc}
\hline No. & BH-NS formation scenario & $\mathcal{R}_{\text {M480.B }}$ & $\mathcal{R}_{\text {M481.B }}$ & $\mathcal{R}_{\text {M380.B }}$ \\
\hline \hline 1. & MT1(1/2/4-1) BH CE2(14-4/5:14-7/8/14) MT2(14-8/9) NS & $\mathbf{1 1 . 6}$ & 0.7 & $\mathbf{6 . 6}$ \\
2. & MT1(1/2/4/5-1/4)/MT2(7-4) BH MT2(14-2/4/5/7/8) NS & 1.2 & 0.0 & 0.0 \\
3. & MT1(1/2/4-1) BH CE2(14-4/5:14-7/8/14) NS & 1.0 & 0.8 & 3.5 \\
4. & MT1(1/2/4-1) BH CE2(14-4/5:14-7/8) MT2(14-8) SW CE2(14-9:14-13) NS & 0.0 & $\mathbf{1 . 5}$ & 0.0 \\
5. & Other scenarios & 1.8 & 1.1 & 3.0 \\
& Total & 15.6 & 4.1 & 13.1 \\
\hline
\end{tabular}

Abrrevations are the analogous with the described in Table 4.

mass mergers and the shape of BH-BH mergers mass distribution.

We present the results of three models; our standard StarTrack input physics model with delayed SN engine (M380.B) and two models with revised RLOF treatment (M480.B and M481.B). Model M480.B differs from our standard M380.B model only by the revised CE development criteria while M481.B additionally includes the modified condition for the switch from TTMT to nuclear timescale MT.

Various changes related to assumptions about RLOF physics may strongly affect (sometimes in a non-intuitive way) the formation of DCO mergers: impacting merger rates and physical properties of merging objects. For the three presented models not only different local merger rate densities or BH mergers mass distributions were obtained but also the dominant evolutionary scenarios for $\mathrm{BH}-\mathrm{BH}$ formation changes.

In model M480.B (with revised CE development criteria) the most common scenario for BH-BH merger formation is without any CE phase. In model M481.B (with revised CE development criteria and modified switch from TTMT to nuclear timescale MT) $\mathrm{BH}-\mathrm{BH}$ dominant formation scenario involves $\mathrm{CE}$ that is preceded by short TTMT phase.

Among the tested models, we did not find one that would be fully consistent with the merger rates for NS-NS, BH-NS, and $\mathrm{BH}-\mathrm{BH}$ given by LIGO/Virgo and at the same time would produce the reported more massive $\mathrm{BH}$ mass distribution for $\mathrm{BH}$ $\mathrm{BH}$ mergers. While the shape of the more massive $\mathrm{BH}$ mass distribution in model M480.B fits well two exponent values and the break point given (The LIGO Scientific Collaboration et al. 2020 ), the total rates for $\mathrm{BH}-\mathrm{BH}$ mergers are too high by the factor of $\sim 2-3$. Additionally, the fraction of unequal BH-BH mergers is not high enough. For Model M481.B the total local rate density for all types of DCO mergers are well consistent with the most recent LIGO/Virgo estimates, but the shape of the more massive BH distribution seems too steep. Obviously, we could try to modify RLOF physics further or even change other parts of input physics (e.g., increase $\mathrm{BH}$ natal kicks to lower BH-BH merger rates) to fit $\mathrm{LIGO/Virgo}$ data for some models. However, the point of this paper was only to demonstrate how uncertain ingredients of population synthesis codes may dramatically alter predictions.

We checked that the fraction of accreted mass during nonconservative RLOF (non-degenerate accretors, see \$2) does not significantly influence the population of BH-BH and BH-NS mergers in tested model M380.B. Beside standard Startrack value $f_{\mathrm{a}}=0.5$, we tested models with values $f_{\mathrm{a}}=0.8$ and $f_{\mathrm{a}}=0.2$ for accreted mass fraction (the fraction $1-f_{\mathrm{a}}$ of transferred mass is ejected from the system). We found that merger rates for $\mathrm{BH}-\mathrm{BH}$ and $\mathrm{BH}-\mathrm{NS}$ systems change about $3 \%$ for $f_{\mathrm{a}}=0.8$ and about $20 \%$ for $f_{\mathrm{a}}=0.2$ comparing to the model with $f_{\mathrm{a}}=0.5$. Also mass and mass ratio distribution for three tested values are similar, with $m_{1}$ (more massive of merging components) slightly shifted to the lower mass values in the model with the lowest accretion fraction $f_{a}=0.2$. Different adopted values of $f_{a}$ influence, however, the local merger rate density for NSNS systems. In both $f_{a}=0.2$ and $f_{a}=0.8$ models the rate for NS-NS systems decreased by a factor of $\sim 2$ comparing to the standard $f_{a}=0.5$ model. The drop in NS-NS rates is a complex mix of multiple overlapping physical processes, e.g., different amount of lost mass (together with orbital angular momentum) leads to different system separation after the first RLOF, which further leads to a different evolutionary type of the donor during the second RLOF (CE phase with HG donor ends with a system merger).

In previous works based on StarTrack code, the two main variants of $\mathrm{CE}$ treatment were usually considered, referred to as submodel A and B. Submodel B (the one presented in this work, see \$2) assumes that all HG donor systems merge during the CE phase. In contrast, submodel A let such binary systems survive if the energy budget calculations allow. We decided to present only the results for the submodel B for two main reasons. First, we checked that typically HG donors which initiate CE in StarTrack are only partially expanded stars, shortly after their main sequence. Such stars likely do not have a clear core-envelope boundary, and therefore, it seems more physical to assume their merger (as we do for main sequence stars). Second, the merger rates for DCOs, especially $\mathrm{BH}-\mathrm{BH}$ systems in submodel A (see Belczynski et al. (2020)) are often much larger (up to 30 times) than estimated by LIGO/Virgo.

Our rapid population synthesis study's central message is that RLOF treatment's choice produces noticeably different populations of GW sources. Our results highlight the need for caution in information inference from stellar and binary evolution models as applied to LIGO/Virgo results. Similar cautionary notes are also found through more detailed evolutionary calculations with hydrodynamical stellar codes (Klencki et al. 2021, Decin 2020). Suppose a population synthesis study is performed with variations of only natal kicks and $\mathrm{CE}$ efficiency, and the match to LIGO/Virgo rates is achieved for some specific CE efficiency and natal kicks assumption. In that case, it cannot be expected to mean that we have already constrained these uncertain parts of input physics.

Acknowledgements. We would like to thank anonymous referee for their useful comments. K.B. and A.O. acknowledge support from the Polish National Science Center (NCN) grant Maestro (2018/30/A/ST9/00050). N.I. acknowledges 
support from CRC program and funding from NSERC Discovery under Grant No. NSERC RGPIN-2019-04277.

\section{References}

Abbott, B., Abbott, R., Abbott, T., et al. 2019, Phys. Rev. X, 9

Abbott, B. P., Abbott, R., Abbott, T. D., et al. 2019a, ApJ, 882, L24

Abbott, B. P., Abbott, R., Abbott, T. D., et al. 2019b, Phys. Rev. X, 9, 031040

Abbott, R., Abbott, T. D., Abraham, S., et al. 2020a, ApJ, 896, L44

Abbott, R., Abbott, T. D., Abraham, S., et al. 2020b, Phys. Rev. Lett., 125, 101102

Andrews, J. J., Cronin, J., Kalogera, V., Berry, C., \& Zezas, A. 2020, arXiv eprints, arXiv:2011.13918

Antonini, F. \& Perets, H. B. 2012a, ApJ, 757, 27

Antonini, F. \& Perets, H. B. 2012b, ApJ, 757, 27

Antonini, F., Toonen, S., \& Hamers, A. S. 2017, ApJ, 841, 77

Arca-Sedda, M. \& Capuzzo-Dolcetta, R. 2019, MNRAS, 483, 152

Arca-Sedda, M., Li, G., \& Kocsis, B. 2018, arXiv e-prints, arXiv:1805.06458

Askar, A., Szkudlarek, M., Gondek-Rosińska, D., Giersz, M., \& Bulik, T. 2017, MNRAS, 464, L36

Bae, Y.-B., Kim, C., \& Lee, H. M. 2014, MNRAS, 440, 2714

Banerjee, S. 2018, MNRAS, 473, 909

Bavera, S. S., Fragos, T., Qin, Y., et al. 2020, A\&A, 635, A97

Begelman, M. C. 1979, MNRAS, 187, 237

Belczynski, K. 2020, ApJ, 905, L15

Belczynski, K., Dominik, M., Bulik, T., et al. 2010, ApJ, 715, L138

Belczynski, K., Heger, A., Gladysz, W., et al. 2016a, A\&A, 594, A97

Belczynski, K., Holz, D. E., Bulik, T., \& O'Shaughnessy, R. 2016b, Nature, 534, 512

Belczynski, K., Kalogera, V., \& Bulik, T. 2002, ApJ, 572, 407

Belczynski, K., Kalogera, V., Rasio, F. A., et al. 2008, ApJS, 174, 223

Belczynski, K., Klencki, J., Fields, C. E., et al. 2020, A\&A, 636, A104

Belczynski, K., Taam, R. E., Kalogera, V., Rasio, F. A., \& Bulik, T. 2007, ApJ, 662,504

Belczynski, K., Wiktorowicz, G., Fryer, C. L., Holz, D. E., \& Kalogera, V. 2012, ApJ, 757, 91

Benacquista, M. J. \& Downing, J. M. B. 2013, Living Reviews in Relativity, 16, 4

Bethe, H. A. \& Brown, G. E. 1998, The Astrophysical Journal, 506, 780-789

Bond, J. R. \& Carr, B. J. 1984, MNRAS, 207, 585

Broekgaarden, F. S., Berger, E., Neijssel, C. J., et al. 2021, arXiv e-prints, arXiv:2103.02608

Chatterjee, S., Rodriguez, C. L., Kalogera, V., \& Rasio, F. A. 2017, ApJ, 836, L26

Cherepashchuk, A. M., Postnov, K. A., \& Belinski, A. A. 2019, MNRAS, 485, 2638

Chruślińska, M., Jeřábková, T., Nelemans, G., \& Yan, Z. 2020, A\&A, 636, A10

Clayton, M., Podsiadlowski, P., Ivanova, N., \& Justham, S. 2017, MNRAS, 470, 1788

de Kool, M. 1990, ApJ, 358, 189

de Mink, S. E. \& Mandel, I. 2016, MNRAS, 460, 3545

Decin, L. 2020, arXiv e-prints, arXiv:2011.13472

Dewi, J. D. M. \& Tauris, T. M. 2000, A\&A, 360, 1043

Di Carlo, U. N., Giacobbo, N., Mapelli, M., et al. 2019, MNRAS, 487, 2947

Dominik, M., Belczynski, K., Fryer, C., et al. 2012, ApJ, 759, 52

Downing, J. M. B., Benacquista, M. J., Giersz, M., \& Spurzem, R. 2010, MNRAS, 407, 1946

Drozda, P., Belczynski, K., O’Shaughnessy, R., Bulik, T., \& Fryer, C. L. 2020, arXiv e-prints, arXiv:2009.06655

du Buisson, L., Marchant, P., Podsiadlowski, P., et al. 2020, MNRAS, 499, 5941

Eggleton, P. P. 2002, ApJ, 575, 1037

Eldridge, J. J. \& Stanway, E. R. 2016, MNRAS, 462, 3302

Fishbach, M. \& Holz, D. E. 2020, ApJ, 891, L27

Fragione, G., Grishin, E., Leigh, N. W. C., Perets, H. B., \& Perna, R. 2019, MNRAS, 488, 47

Fragione, G. \& Kocsis, B. 2019, MNRAS, 486, 4781

Fragos, T., Andrews, J. J., Ramirez-Ruiz, E., et al. 2019, ApJ, 883, L45

Fryer, C. L., Belczynski, K., Wiktorowicz, G., et al. 2012, ApJ, 749, 91

Ge, H., Hjellming, M. S., Webbink, R. F., Chen, X., \& Han, Z. 2010, ApJ, 717, 724

Ge, H., Webbink, R. F., Chen, X., \& Han, Z. 2015, ApJ, 812, 40

Ge, H., Webbink, R. F., Chen, X., \& Han, Z. 2020a, ApJ, 899, 132

Ge, H., Webbink, R. F., \& Han, Z. 2020b, ApJS, 249, 9

Grichener, A. \& Soker, N. 2021, arXiv e-prints, arXiv:2101.05118

Gültekin, K., Miller, M. C., \& Hamilton, D. P. 2004, ApJ, 616, 221

Gültekin, K., Miller, M. C., \& Hamilton, D. P. 2006, ApJ, 640, 156

Hainich, R., Oskinova, L. M., Shenar, T., et al. 2018, A\&A, 609, A94

Hamers, A. S., Bar-Or, B., Petrovich, C., \& Antonini, F. 2018, ApJ, 865, 2
Han, Z., Podsiadlowski, P., \& Eggleton, P. P. 1994, MNRAS, 270, 121

Han, Z.-W., Ge, H.-W., Chen, X.-F., \& Chen, H.-L. 2020, RAA, 20, 161

Hartwig, T., Volonteri, M., Bromm, V., et al. 2016, MNRAS, 460, L74

Hoang, B.-M., Naoz, S., Kocsis, B., Rasio, F. A., \& Dosopoulou, F. 2018, ApJ, 856,140

Hobbs, G., Lorimer, D. R., Lyne, A. G., \& Kramer, M. 2005, MNRAS, 360, 974

Horvath, J. E., Rocha, L. S., Bernardo, A. L. C., de Avellar, M. G. B., \& Valentim, R. 2020, arXiv e-prints, arXiv:2011.08157

Howitt, G., Stevenson, S., Vigna-Gómez, A., et al. 2020, MNRAS, 492, 3229

Hurley, J. R., Pols, O. R., \& Tout, C. A. 2000, MNRAS, 315, 543

Hurley, J. R., Sippel, A. C., Tout, C. A., \& Aarseth, S. J. 2016, MNRAS, 33, e036

Hurley, J. R., Tout, C. A., \& Pols, O. R. 2002, MNRAS, 329, 897-928

Iaconi, R. \& De Marco, O. 2019, MNRAS, 490, 2550-2566

Iaconi, R., Reichardt, T., Staff, J., et al. 2017, MNRAS, 464, 4028

Ivanova, N., Justham, S., \& Ricker, P. 2020, Common Envelope Evolution, 25143433 (IOP Publishing)

Ivanova, N. \& Taam, R. E. 2004, ApJ, 601, 1058

Jones, D. 2020, Reviews in Frontiers of Modern Astrophysics, 123-153

Kalogera, V. \& Webbink, R. F. 1996, ApJ, 458, 301

King, A. R., Davies, M. B., Ward, M. J., Fabbiano, G., \& Elvis, M. 2001, ApJ, 552, L109

Kinugawa, T., Inayoshi, K., Hotokezaka, K., Nakauchi, D., \& Nakamura, T. 2014, MNRAS, 442, 2963

Klencki, J., Nelemans, G., Istrate, A. G., \& Chruslinska, M. 2021, A\&A, 645, A54

Kremer, K., Ye, C. S., Rui, N. Z., et al. 2020, ApJS, 247, 48

Kroupa, P. 2002, Science, 295, 82

Kroupa, P., Tout, C. A., \& Gilmore, G. 1993, MNRAS, 262, 545

Kruckow, M. U., Tauris, T. M., Langer, N., Kramer, M., \& Izzard, R. G. 2018, MNRAS, 481, 1908

Lai, D., Rasio, F. A., \& Shapiro, S. L. 1993, ApJS, 88, 205

Leiner, E. M. \& Geller, A. 2021, ApJ, 908, 229

Lipunov, V. M., Postnov, K. A., \& Prokhorov, M. E. 1997, Astron. Lett., 23, 492

Liu, B. \& Lai, D. 2018, ApJ, 863, 68

Livio, M. \& Soker, N. 1988, ApJ, 329, 764

MacLeod, M., Antoni, A., Murguia-Berthier, A., Macias, P., \& Ramirez-Ruiz, E. 2017, ApJ, 838, 56

MacLeod, M. \& Ramirez-Ruiz, E. 2015, ApJ, 803, 41

Mandel, I. \& de Mink, S. E. 2016, MNRAS, 458, 2634

Mandel, I., Müller, B., Riley, J., et al. 2021, MNRAS, 500, 1380

Mapelli, M. 2016, MNRAS, 459, 3432

Marchant, P., Langer, N., Podsiadlowski, P., Tauris, T. M., \& Moriya, T. J. 2016, A\&A, 588, A50

Marchant, P., Pappas, K. M. W., Gallegos-Garcia, M., et al. 2021, arXiv e-prints, arXiv:2103.09243

Marchant, P., Renzo, M., Farmer, R., et al. 2019, ApJ, 882, 36

Mennekens, N. \& Vanbeveren, D. 2014, A\&A, 564, A134

Meurs, E. J. A. \& van den Heuvel, E. P. J. 1989, A\&A, 226, 88

Miller, M. C. \& Hamilton, D. P. 2002a, ApJ, 576, 894

Miller, M. C. \& Hamilton, D. P. 2002b, MNRAS, 330, 232

Misra, D., Fragos, T., Tauris, T. M., Zapartas, E., \& Aguilera-Dena, D. R. 2020, A\&A, 642, A174

Mondal, S., Belczyński, K., Wiktorowicz, G., Lasota, J.-P., \& King, A. R. 2020, MNRAS, 491, 2747

Morawski, J., Giersz, M., Askar, A., \& Belczynski, K. 2018, MNRAS, 481, 2168

Nandez, J. L. A. \& Ivanova, N. 2016, MNRAS, 460, 3992

Nandez, J. L. A., Ivanova, N., \& Lombardi, J. C. J. 2015, MNRAS, 450, L39

O'Leary, R. M., O'Shaughnessy, R., \& Rasio, F. A. 2007, Phys. Rev. D, 76, 061504

Olejak, A., Fishbach, M., Belczynski, K., et al. 2020, ApJ, 901, L39

Paczynski, B. 1976, in IAU Symposium, Vol. 73, Structure and Evolution of Close Binary Systems, ed. P. Eggleton, S. Mitton, \& J. Whelan, 75

Paczyński, B. \& Ziółkowski, J. 1968, Acta Astron., 18, 255

Passy, J.-C., De Marco, O., Fryer, C. L., et al. 2012, ApJ, 744, 52

Pavlovskii, K., Ivanova, N., Belczynski, K., \& Van, K. X. 2017, MNRAS, 465, 2092

Perna, R., Wang, Y.-H., Farr, W. M., Leigh, N., \& Cantiello, M. 2019, ApJ, 878, L1

Peters, P. C. 1964, PhD thesis, California Institute of Technology

Podsiadlowski, P., Ivanova, N., Justham, S., \& Rappaport, S. 2010, MNRAS, 406, 840

Portegies Zwart, S. F., Baumgardt, H., Hut, P., Makino, J., \& McMillan, S. L. W. 2004, Nature, 428, 724

Portegies Zwart, S. F. \& Verbunt, F. 1996, A\&A, 309, 179

Ricker, P. M. \& Taam, R. E. 2008, ApJ, 672, L41

Rodriguez, C. L., Amaro-Seoane, P., Chatterjee, S., et al. 2018, Phys. Rev. D, 98, 123005

Rodriguez, C. L., Haster, C.-J., Chatterjee, S., Kalogera, V., \& Rasio, F. A. 2016, ApJ, 824, L8 
Sadowski, A., Belczynski, K., Bulik, T., et al. 2008, ApJ, 676, 1162

Sand, C., Ohlmann, S. T., Schneider, F. R. N., Pakmor, R., \& Röpke, F. K. 2020 , A\&A, 644, A60

Santoliquido, F., Mapelli, M., Bouffanais, Y., et al. 2020, ApJ, 898, 152

Santoliquido, F., Mapelli, M., Giacobbo, N., Bouffanais, Y., \& Artale, M. C. 2021, MNRAS, 502, 4877

Shiber, S., Iaconi, R., De Marco, O., \& Soker, N. 2019, MNRAS, 488, 5615

Silsbee, K. \& Tremaine, S. 2017, ApJ, 836, 39

Spera, M., Giacobbo, N., \& Mapelli, M. 2016, Mem. Soc. Astron. Italiana, 87, 575

Spera, M., Mapelli, M., Giacobbo, N., et al. 2019, MNRAS, 485, 889

Stevenson, S., Vigna-Gómez, A., Mandel, I., et al. 2017, Nat. Commun, 8, 14906

Tanikawa, A., Susa, H., Yoshida, T., Trani, A. A., \& Kinugawa, T. 2021, ApJ, 910,30

The LIGO Scientific Collaboration \& the Virgo Collaboration. 2020, arXiv eprints, arXiv:2004.08342

The LIGO Scientific Collaboration, the Virgo Collaboration, Abbott, R., et al. 2020, arXiv e-prints, arXiv:2010.14533

Tutukov, A. V. \& Yungelson, L. R. 1993, MNRAS, 260, 675

van den Heuvel, E. P. J. 1976, in IAU Symposium, Vol. 73, Structure and Evolution of Close Binary Systems, ed. P. Eggleton, S. Mitton, \& J. Whelan, 35

van den Heuvel, E. P. J., Portegies Zwart, S. F., \& de Mink, S. E. 2017, MNRAS, 471,4256

Vanbeveren, D. 1991, A\&A, 252, 159

Vanbeveren, D., De Donder, E., Van Bever, J., Van Rensbergen, W., \& De Loore, C. 1998, New A, 3, 443

VanLandingham, J. H., Miller, M. C., Hamilton, D. P., \& Richardson, D. C. 2016, ApJ, 828, 77

Vigna-Gómez, A., MacLeod, M., Neijssel, C. J., et al. 2020, PASA, 37, e038

Vinciguerra, S., Neijssel, C. J., Vigna-Gómez, A., et al. 2020, MNRAS, 498, 4705

Vink, J. S., de Koter, A., \& Lamers, H. J. G. L. M. 2001, A\&A, 369, 574

Voss, R. \& Tauris, T. M. 2003, MNRAS, 342, 1169

Webbink, R. F. 1984, ApJ, 277, 355

Woosley, S. E. 2016, ApJ, 824, L10

Woosley, S. E. 2017, ApJ, 836, 244

Xu, X.-J. \& Li, X.-D. 2010, ApJ, 716, 114-121

Zevin, M., Bavera, S. S., Berry, C. P. L., et al. 2020, arXiv e-prints, arXiv:2011.10057

Zevin, M., Samsing, J., Rodriguez, C., Haster, C.-J., \& Ramirez-Ruiz, E. 2019, ApJ, 871, 91

Zuo, Z.-Y. \& Li, X.-D. 2014, MNRAS, 442, 1980-1991 


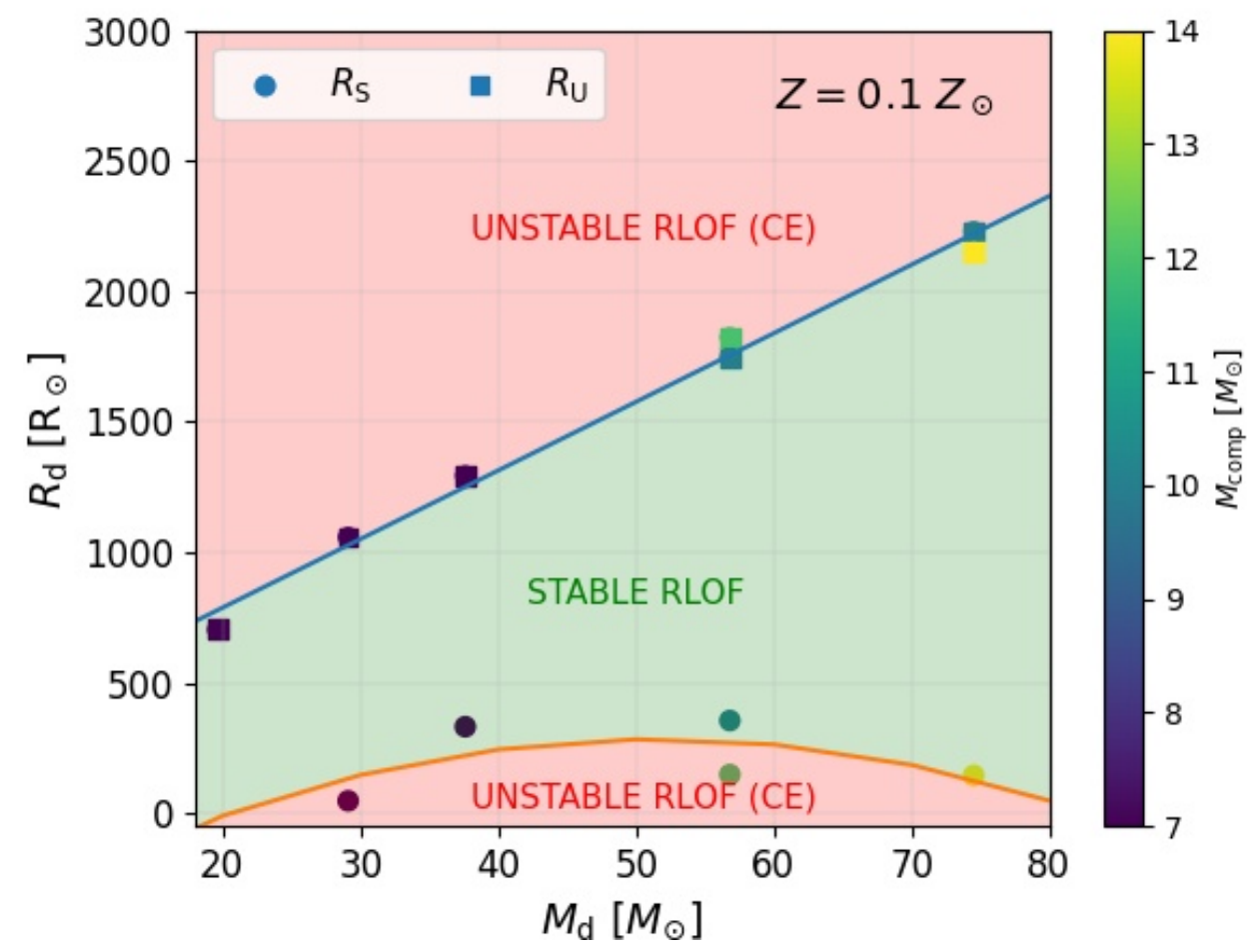

Fig. 2. Revised CE development criteria for stars with $10 \%$ of solar metallicity. On horizontal axis is the mass of the donor star. On the vertical axis is the radius of the donor. The circle and square points mark values of $R_{\mathrm{S}}$ and $R_{\mathrm{U}}$, respectively (see Table 2). We fit lines to these data (see eq. 45 and 6 to show regions of stable RLOF and unstable RLOF (CE). The color coded bar on the right denotes companion mass.

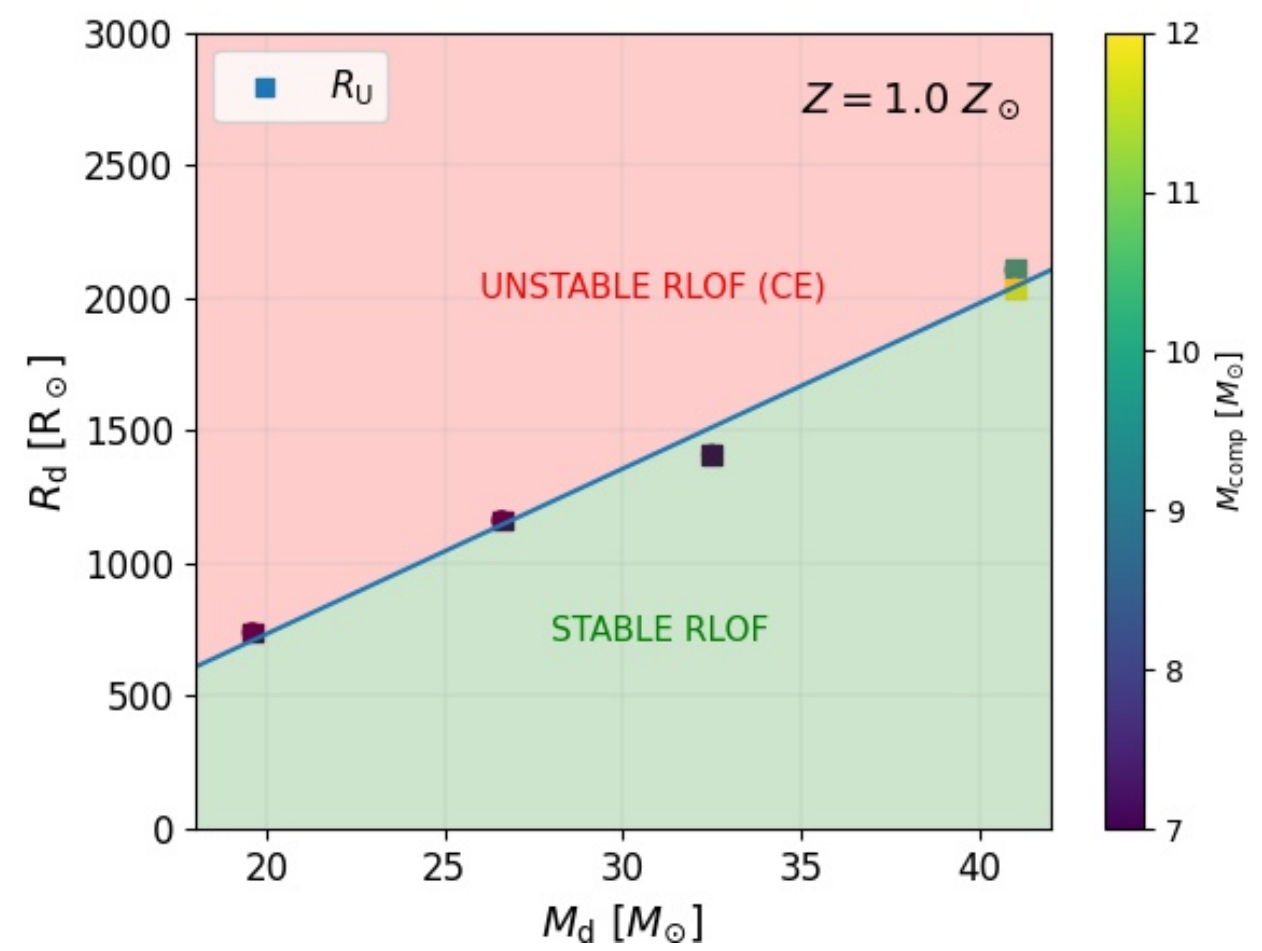

Fig. 3. Revised CE development criteria for stars with $100 \%$ of solar metallicity. Notation is the same as on Figure 2 


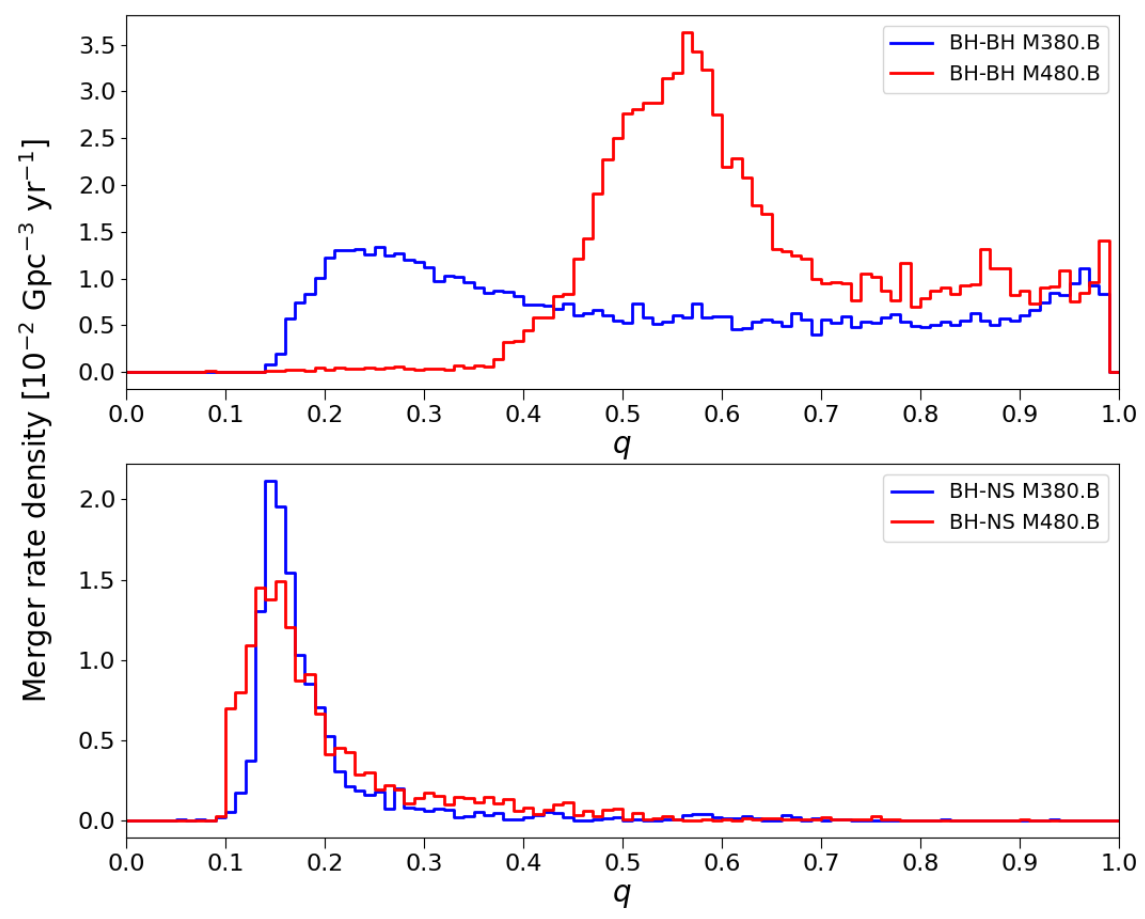

Fig. 4. Distribution of mass ratio in BH-BH and BH-NS mergers $(\mathrm{z} \sim 0)$. On the top panel BH-BH mergers, on the bottom panel BH-NS mergers. Blue line - results for standard CE development criteria with delayed SN engine (M380.B); red line - results for revised CE development criteria (M480.B).

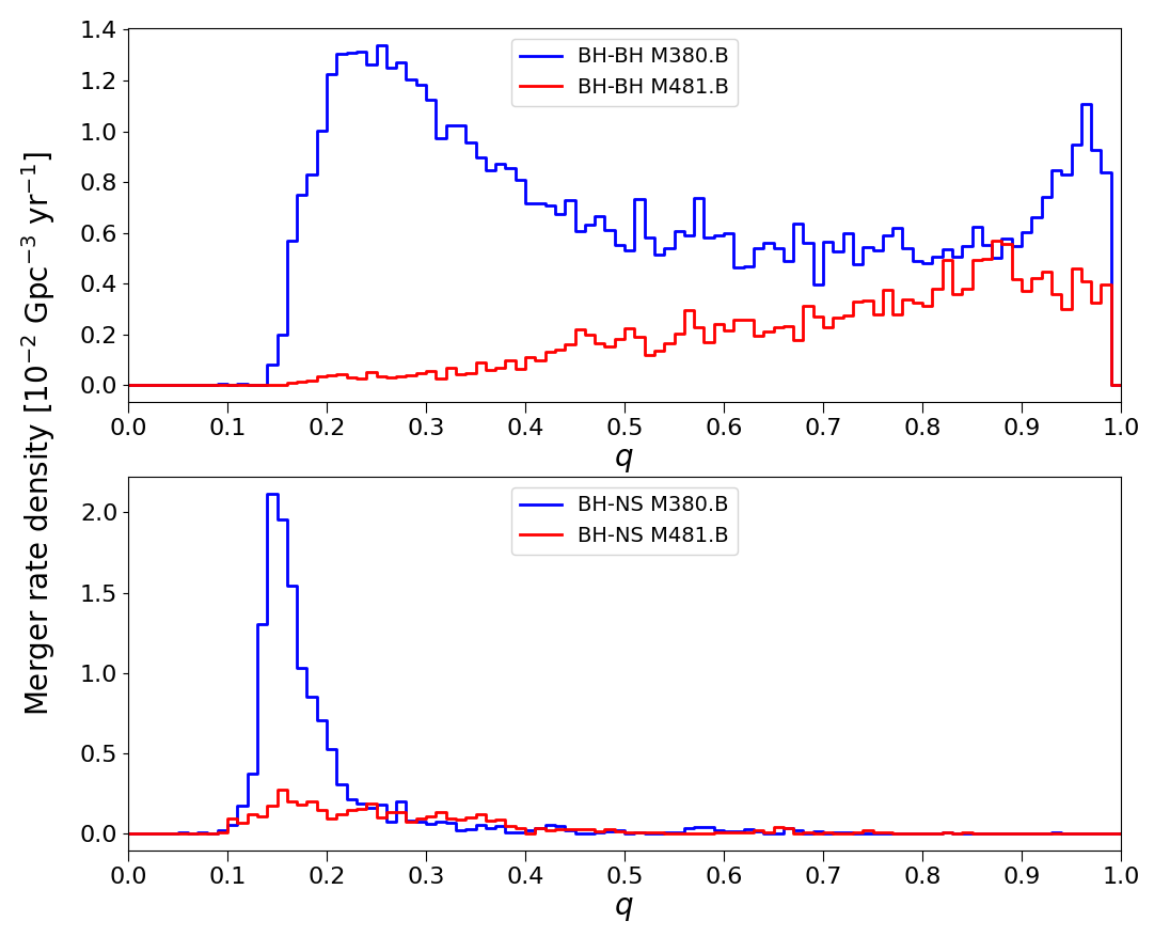

Fig. 5. Distribution of mass ratio in BH-BH and BH-NS mergers $(\mathrm{z} \sim 0)$. On the top panel BH-BH mergers, on the bottom panel BH-NS mergers. Blue line - results for standard CE development criteria with delayed SN engine (M380.B); red line - results for revised CE development criteria and revised stable RLOF treatment (M481.B). 


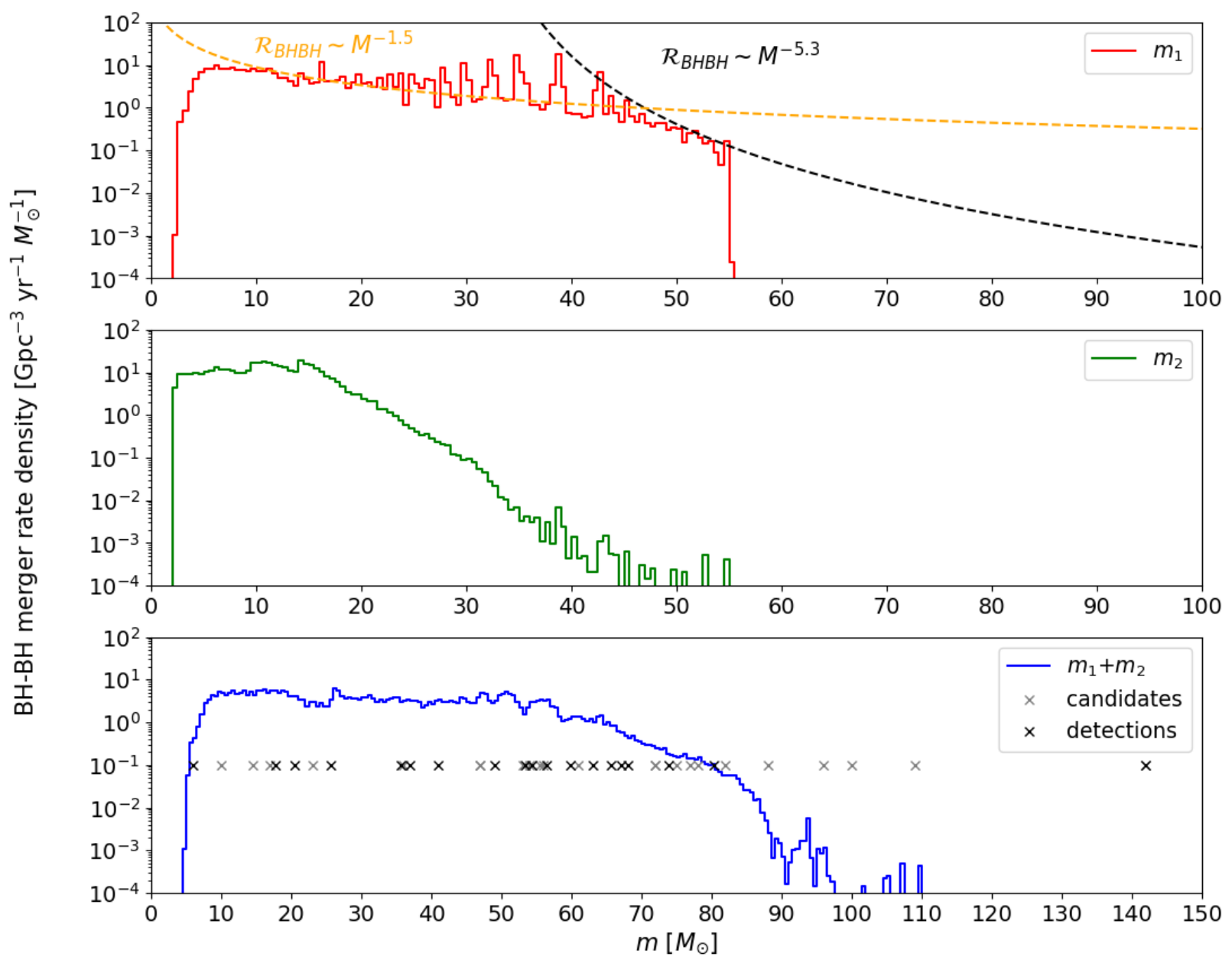

Fig. 6. Intrinsic $\mathrm{BH}-\mathrm{BH}$ merger $(z<2)$ mass distributions: more massive $\mathrm{BH}$ mass (top panel), less massive $\mathrm{BH}$ mass (middle panel), and total mass (bottom panel). We also show total mass estimates for LIGO/Virgo BH-BH mergers from O1, O2 and O3. Results for model M480.B. 

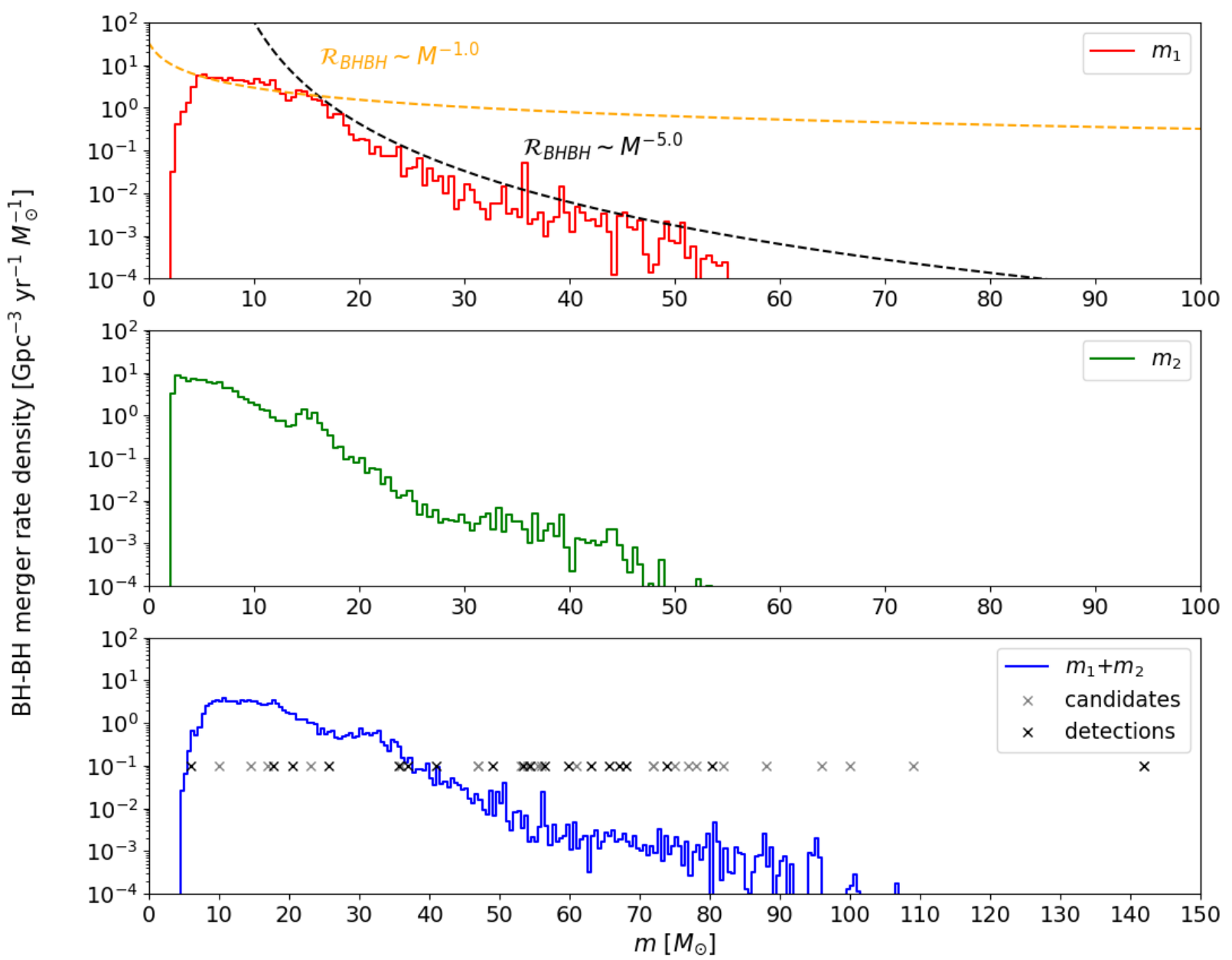

Fig. 7. Intrinsic BH-BH merger $(z<2)$ mass distributions: more massive BH mass (top panel), less massive BH mass (middle panel), and total mass (bottom panel). We also show total mass estimates for LIGO/Virgo BH-BH mergers from O1, O2 and O3. Results for model M481.B. 


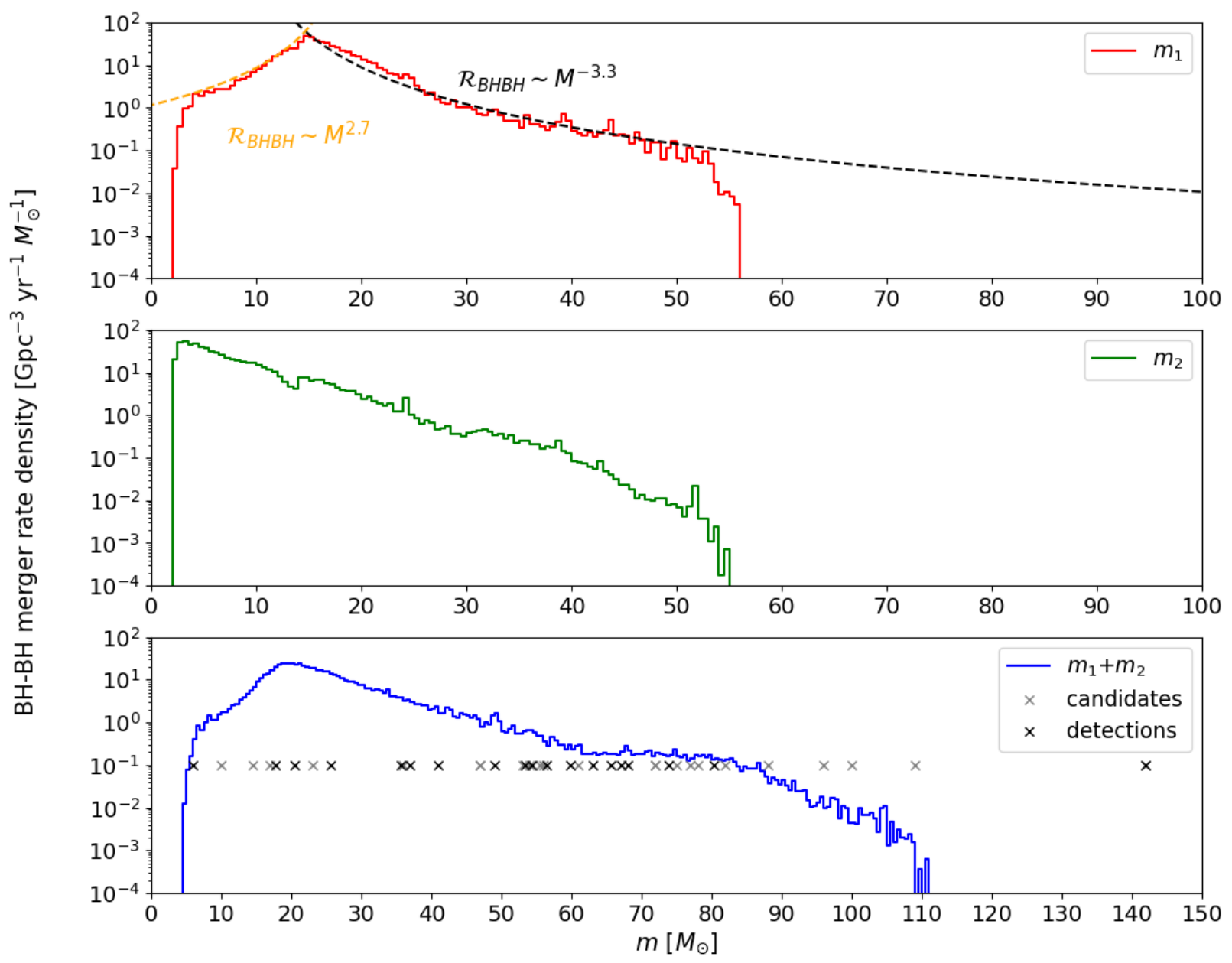

Fig. 8. Intrinsic BH-BH merger $(z<2)$ mass distributions: more massive BH mass (top panel), less massive BH mass (middle panel), and total mass (bottom panel). We also show total mass estimates for LIGO/Virgo BH-BH mergers from O1, O2 and O3. Results for model M380.B (standard). 


\section{Time [Myr]}

$-0.00$

$-4.34$

$-4.84$

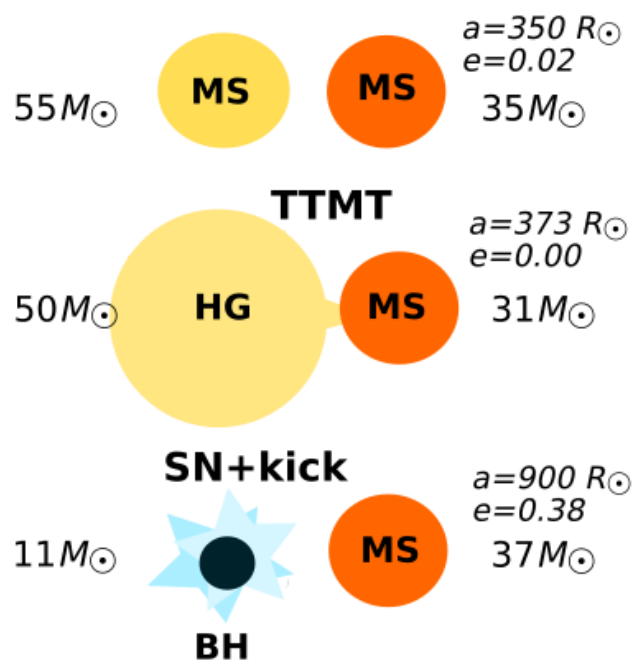

$Z=0.0085$

BH

M380

$-6.50$

$11 M \odot$

CE $a=900 R \odot$ $e=0.00$

HG

BH

BH+HG star merger

$-6.54$

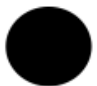

M480

$11 M \odot$

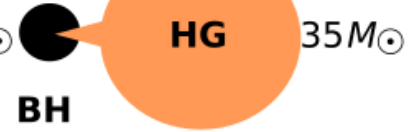

$a=150 R \odot$

$11 M \odot$

BH
He

M481
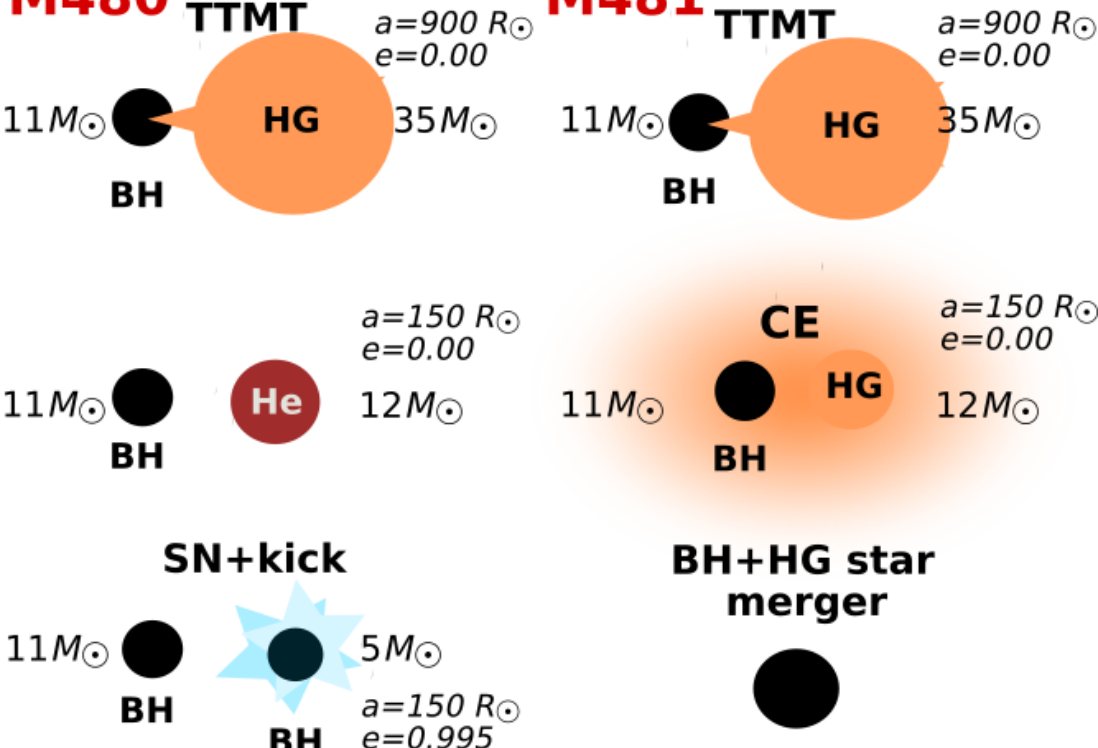

$a=150 R \odot$ $e=0.00$

HG $12 M \odot$
BH

BH+HG star merger

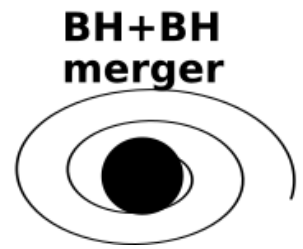

Fig. 9. The typical local $(z \approx 0)$ BH-BH mergers formation scenarios for model M480.B together with the alternative evolution of the same system in models M380.B and M481.B. For all models we begin with the same initial conditions, massive system of $55 M_{\odot}$ primary and $35 M_{\odot}$ secondary on the orbit of $350 R_{\odot}$ and with metallicity $Z=0.0085$. In all scenarios the primary initiates TTMT when it leaves main sequence and begins to expand in thermal timescale during the $\mathrm{HG}$ phase. After $4.8 \mathrm{Myr}$ the primary finishes evolution and explodes as type $\mathrm{Ib} / \mathrm{Ic} \mathrm{SN}$ leaving behind a $11 M_{\odot} \mathrm{BH}$ remnant. Next, after the secondary leaves main sequence, due to more restricted condition for CE development, in the cases of model M480.B and 481.B system goes through TTMT instead of CE phase as in standard M380.B model. During TTMT the secondary looses large percent of its mass (over $60 \%$ ) together with the system orbital angular momentum. Therefore, the orbit tightens (by a factor of $\sim 4$ ). After TTMT, in model 480.B the system remains BH-He system on the orbit of $150 R_{\odot}$. Due to the high natal kick after the second SN explosion, the orbital eccentricity significantly increases to $\mathrm{e}=0.995$ what allows the BH-BH system to merge in Hubble time. In standard (M380.B) scenario systems goes through CE phase with HG donor and we assume system a merger (see \$2). In model M481.B due to modified condition for switch between the TTMT and nuclear-timescale stable MT, and the "safety" condition ( $\operatorname{read} \$ 3.2$ ) after TTMT system also enters CE phase with HG donor and merges leaving behind a single $\mathrm{BH}$. "He" in the diagram stands for a stripped helium core. 


\section{Time [Myr]}

$-0.00$

$-3.25$

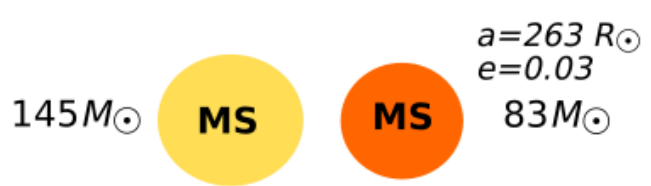

\section{TTMT}

$$
a=256 R \odot
$$

$e=0.00$

M380/M480

$142 \mathrm{M} \odot$ HG $\quad$ MS $83 \mathrm{M} \odot$

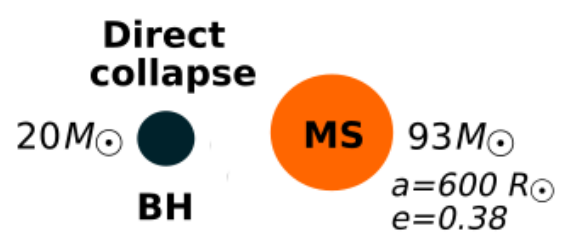

M480

M380
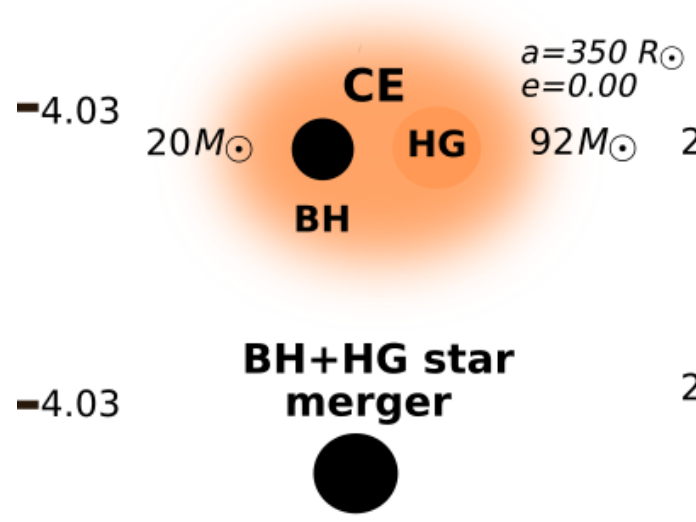

$-4.33$
M481

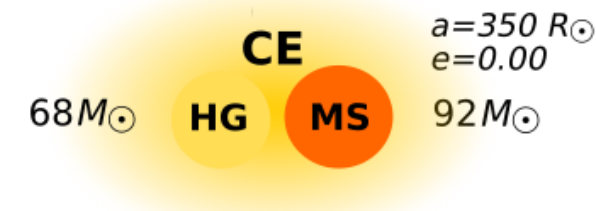

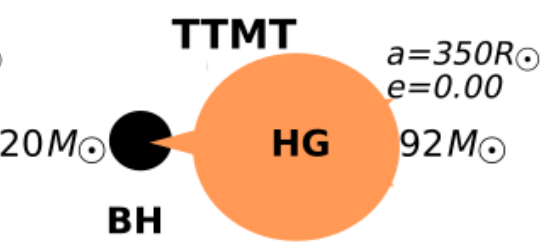

\section{MS star+HG star merger}

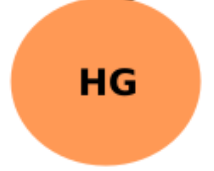

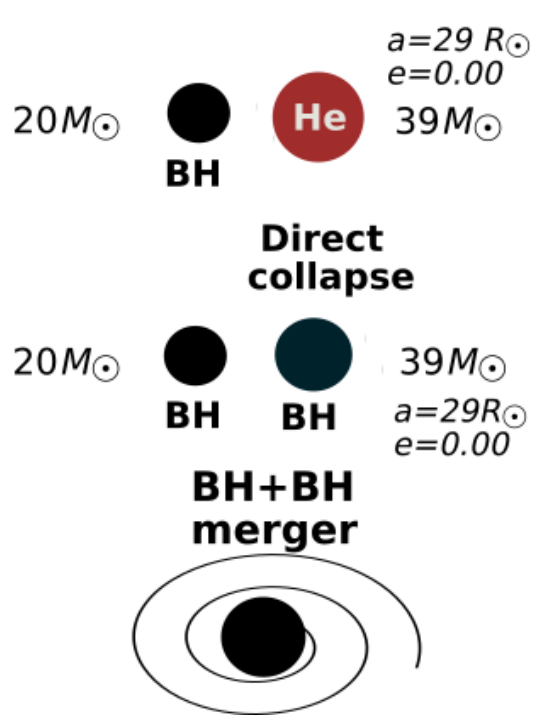

Fig. 10. The typical local $(z \approx 0) \mathrm{BH}-\mathrm{BH}$ mergers formation scenarios for model M480.B together with the alternative evolution of the same system in models M380.B and M481.B. For all models we begin with the same initial conditions, massive system of $145 M_{\odot}$ primary and $83 M_{\odot}$ secondary on the orbit of $263 R_{\odot}$ and with metallicity $Z=0.0001$. In all scenarios the primary initiates TTMT when it leaves main sequence and begins to expand in thermal timescale during the HG phase. When TTMT finishes, in case of models M380.B and M480.B secondary looses envelope. A BH with stripped helium core companion on the orbit of $350 R_{\odot}$ is left. In model M481.B due to modified condition for switch between the TTMT and nuclear-timescale stable MT, and the "safety" condition (read §3.2) when TTMT ends system goes through CE phase. As the donor is HG star, the CE ends with the system merger leaving behind unevolved single star. After $3.3 \mathrm{Myr}$ in models M380.B and M340.B the primary finishes evolution and collapse to a $20 M_{\odot} \mathrm{BH}$ remnant. Next, after the secondary leaves main sequence, due to more restricted condition for CE development, in cases of model M480.B system goes through TTMT instead of CE phase as in standard M380.B model. At the onset of TTMT the secondary (donor) is over 4 times more massive than a BH companion. During the TTMT donor star looses $\sim 60 \%$ of its mass together with the system orbital angular momentum. This leads to the significant orbit tightening by a factor of $\sim 12$. Efficient orbital angular momentum loss during TTMT allows later formed $20 M_{\odot}$ BH and $30 M_{\odot}$ BH system to merge in Hubble time. In the scenario for M380.B highly unequal mass system enters CE phase with HG donor. The binary system does not survive CE phase leaving behind a single $\mathrm{BH}$. "He" in the diagram stands for stripped helium core. 
Time [Myr]

$-0.00$

$-9.12$

$-9.35$

$-27.9$

\section{M380/M480}

$-28.0$

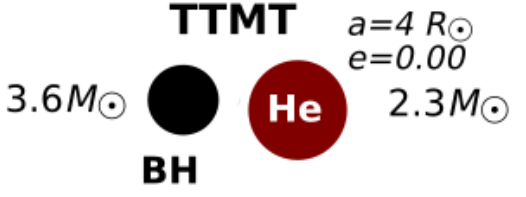

$-28.0$

$-28.0$

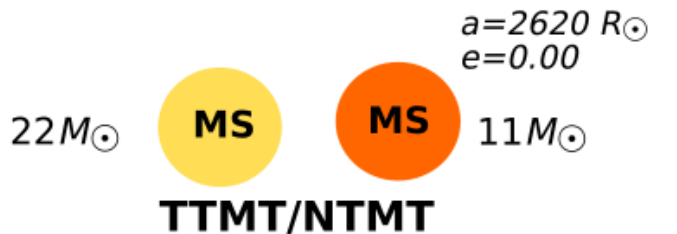

TTMT/NTMT

$16 \mathrm{M} \odot$ СHeB MS $11 \mathrm{M} \odot$

\section{SN+kick}

$3.5 M \odot$

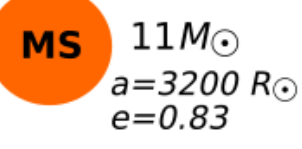

BH
$3.6 M \odot$

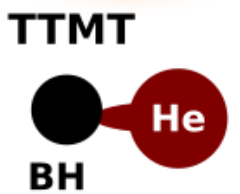

$a=3 R \odot$

$e=0.00$

\section{BH}

$$
\begin{array}{cc}
\text { CE } & \begin{array}{l}
a=970 R \odot \\
e=0.00
\end{array} \\
\text { AGB } & 10.8 \mathrm{M} \odot
\end{array}
$$

